\title{
Die Entwicklung der Steuergerechtigkeitsideen und -prinzipien von Mitte des 17. bis Ende des 18. Jahrhunderts
}

\subsection{Das Zeitalter des Kameralismus}

Für das Abgaben- und Steuerwesen erlangten im 17. Jahrhundert zwei miteinander korrespondierende Prozesse in einer Reihe europäischer Staaten eine maßgebliche Bedeutung. Der eine Prozess war das Entstehen einer neuen Regierungsform, die das feudal-ständestaatliche Ordnungsgefüge ablöste, und der andere Prozess bestand in einer grundlegenden Veränderung der staatlichen Wirtschaftspolitik, indem die vornehmlich seit dem 16. Jahrhundert verfolgte monetaristische Wirtschaftslehre und -praxis durch die Wirtschaftstheorie und Wirtschaftspolitik des Merkantilismus ersetzt wurde. Beide Prozesse setzten Ende des 16. Jahrhunderts ein und kamen im 17. und 18. Jahrhundert vor allem in Frankreich, England, Russland und in einigen Ländern des Heiligen Römischen Reiches Deutscher Nation - in Form des Kameralismus als Sonderform des Merkantilismus - zur Entfaltung. ${ }^{212}$

Mit der Beendigung des Dreißigjährigen Krieges begann eine neue Periode, die bis zu den Auswirkungen der Aufklärungsphilosophie und der Französischen Revolution reichte und mit der Wende vom 18. zum 19. Jahrhundert ausklang. Die wichtigsten Tatsachen, die diese Epoche von der vorhergehenden strukturell unterschieden, waren der Sieg des absoluten Staates über den ständischen Staat und die Einbeziehung der Lenkung der Wirtschaft in den Aufgabenbereich dieses Staates,

212 Sahm, Reiner: 5000 Jahre Steuern, S. 130. 
die damit im Zusammenhang stehende politische Zersplitterung in geschlossene Einzelstaaten und der wirtschaftliche Zusammenbruch im Dreißigjährigen Krieg. In Deutschland erhielten durch die Friedensakte von Münster und Osnabrück (1648) die einzelnen Länder die volle Souveränität zugebilligt. ${ }^{213}$

\subsubsection{Das Entstehen einer neuen Regierungsform}

Die ersten theoretischen Grundlagen einer neuen Regierungsform lieferten Staatstheoretiker. Einer der Ersten war der Franzose Jean Bodin (1530-1596), ${ }^{214}$ der seine Staatstheorie in seinem 1576 unter dem Titel „Six livres de la Republique“ erschienenen Hauptwerk dargelegte. Er beschrieb sein Ideal von einem Staat, der eine absolute und unteilbare Souveränität besitzt und über das alleinige Gesetzgebungsrecht verfügt, um einander befehdende Gruppen zu Ruhe und Ordnung zu zwingen. Das Staatsideal von Bodin betonte die umfassenden Rechte der obersten Gewalt, war aber von den Staatsvorstellungen des voll ausgebildeten Absolutismus noch weit entfernt. ${ }^{215}$

Der Engländer Thomas Hobbes (1588-1679) bereiste mehrfach den europäischen Kontinent und machte sich mit den in den verschiedenen Ländern herrschenden Gesellschaftsverhältnissen vertraut. Im Laufe seiner wissenschaftlichen Betrachtungen kam er - aufbauend auf den Ideen von Bodin - in seinem Meisterwerk „Leviathan“, das 1651 erschien, zu der Erkenntnis, dass der Mensch ein egoistisches Wesen sei, das von grundlegenden Leidenschaften bestimmt werde. Die damit verbundene völlige Anarchie könne - so Hobbes - nicht ausgeschaltet, wohl aber durch instruierende Vernunft gezügelt werden. Im Interesse der Selbsterhaltung könnten die Menschen untereinander Vereinbarungen treffen und sich zur Bändigung ihrer Leidenschaften einem Souverän unterwerfen. Dieser Souverän, gleichgültig ob Monarch, Aristokratie oder Demokratie, stehe in keinem vertraglichen Verhältnis zu den Mitgliedern des so gebildeten Staates und könne deshalb auch nicht von ihnen belangt werden. Die Macht des Souveräns sei unbegrenzt, erstrecke sich auf weltliche und geistige Fragen und sei unteilbar. Allerdings hätten die Gesellschaftsmitglieder das Recht zum Ungehorsam, wenn sich zeige, dass der Souverän nicht mehr imstande sei, Ordnung zu halten und den Schutz des Lebens

213 Lütge, Friedrich: Deutsche Sozial- und Wirtschaftsgeschichte. Ein Überblick, 3. Aufl., Berlin/Heidelberg/New York 1979, S. 322.

214 Vgl. hierzu die Ausführungen in Kapitel 4.1.

215 Herzfeld, Hans (Hrsg.): Geschichte in Gestalten, Bd. 1, Frankfurt am Main 1981, S. 167 f.; Sahm, Reiner: 5000 Jahre Steuern, S. 131. 
der Gesellschaftsmitglieder zu gewährleisten. Sie könnten sich dann einem neuen Souverän unterwerfen. ${ }^{216}$

Es fehlte jedoch nicht an Gegnern, die die Auswüchse dieser Lehre bekämpften und darauf hinwiesen, dass das Naturrecht, das das Privateigentum schütze, auch den Fürsten verpflichte. Der Philosoph John Locke (1632-1704) argumentierte gegen diese Machtfülle; nur dann, wenn das steuerzahlende Volk durch seine Repräsentanten die öffentlichen Bedürfnisse anerkennt und die Steuern gutheißt, könne die Besteuerung gerechtfertigt werden. ${ }^{217}$

Das Charakteristische dieser nun in Erscheinung tretenden absolutistischen Regierungsform war, dass der Monarch die Herrschaftsgewalt in seinem Land allein ausübte und sich bei seinen Entscheidungen nicht an die Zustimmung autonomer politischer Körperschaften gebunden fühlte. Der absolute Fürst erkannte wohl das göttliche und historische Recht an, stand aber selbst über den gegebenen Gesetzen und konnte sie brechen. Er war der einzige Repräsentant der Staatsgewalt, der die unumschränkte Macht als oberster Gesetzgeber, Regierungsoberhaupt, oberster Richter und Militärbefehlshaber besaß. Dieser Machtgedanke schloss in sich die anmaßende Forderung ein, Recht setzen zu können. ${ }^{218}$

Die Entwicklung zu der absolutistischen Staatsform stand im Zusammenhang mit lang währenden militärischen Auseinandersetzungen, die dazu führten, dass sich die ökonomische Leistungsfähigkeit der daran beteiligten Länder allmählich erschöpfte und es einer bestimmten Art und Weise der wirtschaftlichen Rekonstruktion bedurfte. Die Notwendigkeit zu einer solchen Rekonstruktion zwang zu einer Zentralisierung der Möglichkeiten. Das bedingte wiederum eine entsprechende Führung und Organisation, die unter den gegebenen Umständen in den Händen eines Monarchen liegen musste und einen funktionstüchtigen zentralen Staatsapparat voraussetzte. Diese Amtsgewalt umfasste die Beamtenschaft, das stehende Heer sowie die Staatskirche, die auf die Konfession des Monarchen ausgerichtet war. Die herausragende Position der Obrigkeit wurde zumeist von einem großen, mit erheblichem finanziellen, materiell-technischen und geistig-kulturellen Aufwand verbundenen Repräsentationsbedürfnis begleitet. ${ }^{219}$

216 Ebd., Bd. 2, S. 212-214; Sahm, Reiner: 5000 Jahre Steuern, S. 131.

217 Mann, Fritz Karl: Steuerpolitische Ideale, S. 19.

218 Lütge, Friedrich: Deutsche Sozial- und Wirtschaftsgeschichte, S. 324; Sahm, Reiner: 5000 Jahre Steuern, S. 131. Un roi, une foi, une loi - ein König, ein Glaube, ein Gesetz - unter dieser Devise herrschte Ludwig der XIV. als Sonnenkönig ein halbes Jahrhundert lang und gab entscheidende Impulse für die Entfaltung des Absolutismus in Europa.

219 Sahm, Reiner: 5000 Jahre Steuern, S. $131 \mathrm{f}$. 


\subsubsection{Die Veränderungen der staatlichen Wirtschaftspolitik durch den Kameralismus}

Eine der Grundvoraussetzungen für das Funktionieren des absolutistischen Staates bestand in der Hebung der Wirtschafts- und Steuerkraft des Landes; dazu musste ein staatswirtschaftliches System begründet und funktionstüchtig gehalten werden. Das Herzstück bildete ein leistungsfähiges Finanz- und Steuerwesen, das in der Lage war, das Beamtentum, das stehende Heer und die sonstigen Staatsaufgaben zu finanzieren. ${ }^{220}$

Das Schwergewicht bei den wirtschaftspolitischen Maßnahmen lag auf einer Beeinflussung des Handels mit dem Ziel, eine aktive Handelsbilanz zu erzielen und damit den eigenen Reichtum zu stärken. In Deutschland standen nicht der Handel im Vordergrund, sondern Landwirtschaft und Gewerbe, die es im Zuge der Wiederaufbaumaßnahmen nach dem Dreißigjährigen Krieg zu fördern und zu entwickeln galt. Die Fokussierung auf natürliche Wachstumsvorgänge und die Entwicklung der produktiven Kräfte war das Ziel im deutschen Merkantilismus; hier hat die Bezeichnung „Kameralismus“ im Vordergrund gestanden, abgeleitet von „camera", der fürstlichen Schatzkammer, die es zu bereichern galt. ${ }^{21}$

Ausgangspunkt aller wirtschaftlichen Handlungsstrategien waren die „Nahrungsgeschäfte“, also die Techniken des Produzierens und Herstellens in Landwirtschaft und Gewerbe. Der Boden, die beweglichen Güter und die Arbeitskraft der Untertanen waren demgemäß die Quellen des Reichtums im Staate und dessen „Vermögen“.222

Im auffallenden Unterschied zur Wissenschaft in Westeuropa entwickelten sich die Kameralwissenschaften im 18. Jahrhundert zu einer eigenständigen, auch universitär gepflegten Wissenschaftsdisziplin. Die Kameralwissenschaftler offerierten eine Fülle wirtschaftspolitischer und administrativer Handlungsstrategien, die einen steuerlich abschöpfungsfähigen Reichtum im Land herbeiführen sollten. ${ }^{223}$

220 Lütge, Friedrich: Deutsche Sozial- und Wirtschaftsgeschichte, S. 328; Sahm, Reiner: 5000 Jahre Steuern, S. 132.

221 Lütge, Friedrich: Deutsche Sozial- und Wirtschaftsgeschichte, S. 329; Isenmann, Moritz: Einleitung, in: Isenmann, Moritz (Hrsg.): Merkantilismus. Wiederaufnahme einer Debatte. (Vierteljahresschrift für Sozial- und Wirtschaftsgeschichte, Beiheft 228), Stuttgart 2014, S. 9-21, S. 14.

222 Justi, Johann Heinrich Gottlob von: Grundsätze der Policey-Wissenschaft (1756), 3. Aufl., Göttingen 1782, S. 10-14.

223 Simon, Thomas: Merkantilismus und Kameralismus. Zur Tragfähigkeit des Merkantilismusbegriffs und seiner Abgrenzung zum deutschen „Kameralismus“, in: Isenmann, Moritz (Hrsg): Merkantilismus. Wiederaufnahme einer Debatte (Vierteljahresschrift für Sozial- und Wirtschaftsgeschichte, Beiheft 228), Stuttgart 2014, S. 65-82, S. 75 f. 
Nachdem im unmittelbaren Anschluss an den Dreißigjährigen Krieg die Zahl juristischer Dissertationen zu Steuern von 1650 bis 1689 mit 50 feststellbaren Titeln ihren absoluten Höhepunkt erreicht hatte, nahm die Anzahl von Werken der mehr ertragsorientierten kameralistischen Steuerliteratur seit Ende des 17. Jahrhunderts stark zu. ${ }^{224}$ Die Autoren befassten sich mit der idealen Ausgestaltung des Steuersystems und dem gegenseitigen Verhältnis der einzelnen Steuern. Nach dem Abflauen des „Akzisenstreits“225 kamen nach 1720 als neue Diskussionsschwerpunkte die Durchführung der Besteuerung und die Möglichkeit einer Reform der über Jahrhunderte gewachsenen territorialen Steuerordnungen hinzu. ${ }^{226}$

\subsubsection{Neue Schwerpunkte der Staatsfinanzierung}

Bei den Veränderungen im Steuer- und Abgabewesen kam es in erster Linie darauf an, die Einnahmen auf eine neue Basis zu stellen. Bislang hatten die Anleihen, die die privaten Finanziers den Landesherren für deren unterschiedliche Vorhaben gewährten, eine außerordentlich große Rolle gespielt. Die Verarmung, die durch den Dreißigjährigen Krieg entstanden war, hatte aber dazu geführt, dass in den deutschen Territorien Anleihen auf längere Zeit nur in Maßen aufgenommen werden konnten, die Landesherren mussten also neue Einnahmequellen erschließen. Das war nicht einfach, denn in den zurückliegenden Jahrhunderten war eine Vielzahl von Steuern und Abgaben aus den unterschiedlichsten Gründen und Anlässen eingeführt worden, ohne darauf Rücksicht zu nehmen, wie sie sich auf diejenigen auswirkten, die sie zu entrichten hatten. ${ }^{227}$

Am Beispiel Bayerns und Preußens wird der Anstieg des Anteils der Steuereinnahmen am Gesamtetat der Territorien deutlich. Bayern hatte Mitte des 18. Jahrhunderts einen Etat in Höhe von 3,8 Millionen fl. Davon entfielen 73,7 Prozent auf Steuereinnahmen und 26,3 Prozent auf Abgaben der Bauern und auf Einnahmen aus Regalien. In Preußen betrugen beim Regierungsantritt Friedrichs des Großen

224 Schulz, Hermann: Das System und die Prinzipien der Einkünfte, führt im Quellenund Literaturverzeichnis 395 Quellen aus der kameralwissenschaftlichen Literatur für den Zeitraum von 1600-1835 an. Bei Jenetzky, Johannes: System und Entwicklung des materiellen Steuerrechts in der wissenschaftlichen Literatur des Kameralismus von 1680-1840, dargestellt anhand der gedruckten zeitgenössischen Quellen (Schriften zum Steuerrecht 17), Berlin 1978, finden sich 327 Titel für den Zeitraum 1680-1840; vgl. Schwennicke, Andreas: „Ohne Steuer kein Staat“, S. 19.

225 Vgl. hierzu Kapitel 5.2.2.

226 Schwenicke, Andreas: Ohne Steuer kein Staat, S. 202 f.

227 Sahm, Reiner: 5000 Jahre Steuern, S. 134. 
die reinen Staatseinnahmen 7 Millionen Taler, davon entfielen 3,3 Millionen (= 47 Prozent) auf die Erzielung von Reinerträgen aus den Domänen. In den Jahren ab 1806 betrugen die gesamten Steuereinnahmen ca. 70 Prozent, die aus dem Domänenbesitz ca. 30 Prozent der gesamten Staatseinnahmen. Aus beiden Beispielen kann man ersehen - was ganz allgemein für diese Epoche gilt -, dass die Bedeutung der Steuern laufend größer wurde. ${ }^{228}$

Staatsbildung und Finanzentwicklung gingen Hand in Hand. Mit dem Wachsen der Staatsintervention, dem Vordringen der Geldwirtschaft und der Einführung stehender Heere wuchs der Finanzbedarf vom 17. Jahrhundert an in allen europäischen Ländern; die Steuern gediehen zu einer ständigen Zwangsabgabe. ${ }^{229}$ In der Literatur des 18. Jahrhunderts wurden die aus der ständigen Kriegsrüstung resultierenden Finanzbedürfnisse als hauptsächlicher Grund und zugleich als Rechtfertigung für die dauernde Auferlegung von Steuern angesehen. ${ }^{230}$

Um die finanziellen Mittel nur für die Kriegsführung aufzubringen, wurden von den Monarchen und ihren Regierungen im Wesentlichen die folgenden Methoden angewandt: Vorerst stützte man sich auf einen über einen längeren Zeitraum angesammelten Kriegsschatz. Friedrich II. bestritt die Ausgaben für den Ersten und Zweiten Schlesischen Krieg ausschließlich aus dem Kriegsschatz. Die zweite Methode bestand in der Manipulation der Währung, also der Vermehrung von unterwertigem Geld. Als dritte Methode wurde die Erhebung von Sondersteuern in Form von Kriegssteuern und von Kontributionen praktiziert. Die vierte Methode war die Aufnahme von Anleihen und die fünfte schließlich die Subsidien, die man im Ausland beschaffte..$^{231}$

228 Lütge, Friedrich: Deutsche Sozial- und Wirtschaftsgeschichte, S. 397.

229 Ullmann, Hans-Peter: Der deutsche Steuerstaat, S. 15; Mann, Fritz Karl: Steuerpolitische Ideale, S. 47 und S. 277.

230 Lang, Karl Heinrich von: Historische Entwicklung, S. 203 ff.; Justi, Johann Heinrich Gottlob von: Ausführliche Abhandlung von denen Steuern und Abgaben nach ächten, aus dem Endzweck der bürgerlichen Gesellschaften abfließenden Grundsätzen und zur Wohlfarth der Völker dienlichen Maaßregeln abgefasset, Königsberg/Leipzig 1762, ND Wiesbaden 1977, S.7.

231 Lütge, Friedrich: Deutsche Sozial- und Wirtschaftsgeschichte, S. 399. 


\subsection{Die Ideale des Absolutismus}

\subsubsection{Zur Entwicklung der steuerpolitischen Anschauungen}

Die Spaltung der politischen Entwicklung in eher absolutistisch regierte oder eher ständisch verwaltete Territorien fand ihren Niederschlag in der Steuerrechtsliteratur; es überwog ein herrschaftsstabilisierender Grundton, wobei die Autoren - je nach ihrem politischen Hintergrund - mehr absolutistische oder mehr ständische Positionen vertraten. In den Territorien, in denen der Einfluss der Stände geringer geworden war, wagten sich nur wenige Autoren an diese komplexe und politisch brisante Materie. Diese Grundstabilität der politischen Verhältnisse des 18. Jahrhunderts dürfte ein wesentliches Moment dafür gewesen sein, dass die Frage der Besteuerung nicht zur Keimzelle revolutionärer Ideen in den deutschen Staaten wurde. ${ }^{232}$

Die überwiegende Auffassung in der juristischen Literatur ging davon aus, dass der Adel wegen des Verbots der doppelten Belastung mit Diensten und Steuern steuerfrei zu sein hat. Da der Adel auch vom Handel und Gewerbe ausgeschlossen war, ${ }^{233}$ ergab es sich, dass im 18. Jahrhundert der historisch gewachsene Bestand an Privilegien als Grundlage der Steuerfreiheit im Vordergrund stand. Erst gegen Ende des 18. Jahrhunderts wurde in verstärktem Maße auch die historische Begründung der Steuerfreiheit des Adels infrage gestellt. ${ }^{234}$

Die Privilegierung von Kirchenvermögen und des Klerus blieb über die Mitte des 17. Jahrhunderts hinaus in der Diskussion, wobei gerade protestantische Autoren der Steuerfreiheit der Kirche oft kritisch gegenüberstanden. Dabei berief sich die Literatur auch auf den Gedanken, dass die Kleriker auch Bürger seien und sich daher an den örtlichen Lasten beteiligen müssten. ${ }^{235}$

Die Reform- und Idealsteuerdiskussionen, die Mitte des 17. Jahrhunderts begonnen hatten, wurden im 18. Jahrhundert in zuvor nicht bekannter Weise von intensiven politischen Debatten um die ideale Gestaltung der Steuersysteme fortgeführt. Aus der Rechtfertigung der Besteuerung - der Steuerbegründungslehre - wurden Regeln der Steuerverteilung abgeleitet. Der gemeinsame Grundgedanke der Steuerlehren dieser Zeit, die später unter dem Sammelnamen „Äquivalenztheorie“ zusammen-

232 Schwennicke, Andreas: „Ohne Steuer kein Staat“, $349 \mathrm{f}$.

233 Zur Diskussion des Handelsverbots vgl. Stollberg-Rilinger, Barbara: Handelsgeist und Adelsethos. Zur Diskussion um das Handelsverbot für den deutschen Adel vom 16. bis zum 18. Jahrhundert, in: Zeitschrift für historische Forschung 15, 1988, S. 290 ff.

234 Ebd., S. 318-327.

235 Ebd., S. 328. 
gefasst worden sind, war, dass Leistung und Gegenleistung einander entsprechen müssten. Die Frage lautete: Welcher Preis entsprach den staatlichen Diensten?

In ethischer Hinsicht stellte sich die Frage, welcher Steuerbetrag gerecht war. Die entwickelten Gerechtigkeitspostulate zielten darauf ab, die zwischen den Individuen durchzuführende Steuerverteilung in einem ethisch als befriedigend angesehenen Sinne zu regeln. Dabei ist insbesondere von großer Bedeutung, ob die den Steuerpflichtigen auferlegte „Belastung“ im Vergleich zu der, die ihre Kollegen, Nachbarn etc. zu tragen hatten, als richtig bemessen erschienen. ${ }^{236}$ Die meisten Steuergrundsätze entstanden aus dem Wunsch, wirtschaftlich, sozial und ethisch als nachteilig empfundene Steuerformen und -techniken zu beseitigen und an ihre Stelle bessere zu setzen. ${ }^{237}$ Fast alle monografischen Abhandlungen zu Steuern befassten sich mit der systematischen Ausformulierung eines umfangreichen Kataloges an Steuergrundsätzen. ${ }^{238}$

\subsubsection{Das Ideal der Akzise}

$\mathrm{Ab}$ der Mitte des 17. Jahrhunderts änderte sich die juristische und politische Beurteilung der idealen Steuerlastverteilung innerhalb weniger Jahre. ${ }^{239}$ Die überwältigende Mehrheit sowohl der Praktiker als auch der Theoretiker stimmte im Grundsatz darin überein, dass die Kontributionen (Steuern auf bewegliche und unbewegliche Güter) und Schatzungen (reelle Vermögensteuern) durch Akzisen ergänzt und sogar ersetzt werden sollten. ${ }^{240}$ Der entscheidende Grund für die Popularität der Akzise lag in der - bereits erörterten - Verarmung, die durch den Dreißigjährigen Krieg entstanden war.

Die Ansichten über das, was der Begriff Akzise beschrieb, gingen weit auseinander, ${ }^{241}$ es lässt sich jedoch festhalten, dass man unter Akzise eine überwiegend den Verbrauch und Verkehr treffende Besteuerung verstand; denn mehrfach traf

236 Vgl. dazu die Hinweise bei Schmölders, Günter: Das Gerechtigkeitspostulat in der Besteuerung, in: Finanzarchiv 23 (1963), S. 53-65.

237 Neumark, Fritz: Grundsätze gerechter und ökonomisch rationaler Steuerpolitik, S. 17.

238 Scheer, Christian: Steuerpolitische Ideale, S. 164.

239 Schwennicke, Andreas: „Ohne Steuer kein Staat“, S. 291.

240 Mann, Fritz Karl: Steuerpolitische Ideale, S. 50.

241 Vgl. Wolf, Jürgen Rainer: „... zu Einführung einer Gott wohlgefälligen Gleichheit auf ewig ....' Steuerreformen im Zeitalter des Absolutismus und der Aufklärung, in: Schultz, Uwe (Hrsg.): Mit dem Zehnten fing es an. Eine Kulturgeschichte der Steuer, München 1986, S. 162-173, S. 168. 
sie die Person, den Besitz und die Produktion und wurde wie eine Katastersteuer veranlagt. Die Einführung und Vermehrung der Akzisen lagen sowohl im Interesse breiter Volksschichten, als auch der absoluten Fürsten, und die Gründe für die Akzise sind in stereotyper Weise wiederholt worden, sobald die indirekten gegenüber den direkten Steuern verteidigt werden sollten. ${ }^{242}$

Für die Akzise sprachen viele Gründe: ${ }^{243}$ zunächst ihre fiskalische Ergiebigkeit - der preußische Fiskus z.B. zog aus der Akzise den Vorteil, dass 1806 über 60 Prozent der Steuereinnahmen aus der städtischen Akzise und dem Zoll flossen, und nur 40 Prozent aus der ländlichen Kontribution ${ }^{244}$ - und ihre Unmerklichkeit. Sie war ein „heimlicher Dieb“, weil sie in einem Augenblick gezahlt wurde, in dem der Mensch an einen Genuss oder ein Vergnügen dachte. Außerdem konnte sie einfach erhoben werden und bestrafte die Verschwendung, regte also zur Sparsamkeit an und nahm - so stellte man fest - nur vom erzielten Ergebnis. Verhinderten zuvor die zäh verteidigten Steuerprivilegien von Adel und Geistlichkeit eine gleichmäßige Lastenverteilung, galt bei bei der Akzise: Wer verbrauchte oder verzehrte, wurde steuerpflichtig, gleichgültig, welchem Stande er angehörte. Auch aus verfassungspolitischen Gründen war die Akzise den Herrschern des 16. bis 18. Jahrhunderts willkommen, denn einmal eingeführte Verbrauchsabgaben konnten ohne neue Zustimmung der Stände fortgeführt werden. Damit verstärkte die Akzise die finanzielle Unabhängigkeit der Fürsten und damit deren Macht.

Die Hervorhebung der Akzise als ideale Steuerform - die durch massive Interessen wirtschaftlicher und politischer Art Unterstützung fand - führte zwangsläufig zu heftigen Gegenbewegungen. Es war deshalb kein Wunder, dass in mehreren Ländern fast gleichzeitig der sogenannte Akzisestreit ausbrach. Die meisten Autoren des 18. Jahrhunderts nahmen Anstoß an der Besteuerung der Grundnahrungsmittel. ${ }^{245}$ Die Kritik richtete sich primär gegen die vorgeschlagene Mehlakzise, denn „die Armen würden durch die aus der Mehlaccise hervorgehende Vertheuerung des Brodes, das ja ihr fast ausschließliches Nahrungsmittel sei, zu Rebellion oder zur Auswanderung gezwungen. ${ }^{\text {"246 }}$

242 Mann, Fritz Karl: Steuerpolitische Ideale, S. $51 \mathrm{f}$.

243 Ebd., S. 53-58.

244 Schmoller, Gustav von: Skizze einer Finanzgeschichte von Frankreich, Österreich, England und Preußen (1500-1900). Historische Betrachtungen über Staatenbildung und Finanzentwicklung, Sonderabdruck aus: Jahrbuch für Gesetzgebung, Verwaltung und Volkswirtschaft im Deutschen Reich 33 (1909), S. 1-64, S. 46.

245 Schwennicke, Andreas: „Ohne Steuer kein Staat“, S. 314.

246 Zitiert in: Inama-Sternegg, Karl Theodor Ferdinand Michael von: Der Accisenstreit deutscher Finanztheoretiker im 17. und 18. Jahrhundert, in: Zeitschrift für die gesammte Staatswissenschaft 21 (1865), S. 515-545, S. 532. 
Mit der literarischen Auseinandersetzung über die Akzise wurde zum ersten Mal in der breiten Öffentlichkeit diskutiert, in welcher Art welche Bevölkerungsgruppen zur Finanzierung der Staatsaufgaben herangezogen werden sollten. ${ }^{247}$ Dieser Streit hatte maßgebenden Einfluss auf die theoretischen Erörterungen über indirekte und direkte Steuern ${ }^{248}$ und auf die Steuersysteme der verschiedenen deutschen Staaten. Er hat die Fürsten, das Beamtentum und den Bürger- und Bauernstand insofern zusammengeschlossen, als die Verbrauchsteuern die Ausnahme von Adel und Geistlichkeit durchbrachen und wenigstens eine teilweise Allgemeinheit der Steuerpflicht verwirklichten. ${ }^{249}$

\subsection{Die Ideale des Naturrechts}

\subsubsection{Die Ansätze einer Naturrechtslehre der Besteuerung}

In keinem Bereich gelangte der Absolutismus schneller an seine Grenzen als in der Finanzwirtschaft. ${ }^{250}$ Alle notwendigen Voraussetzungen für ein geordnetes und ergiebiges Abgabesystem fehlten, und das Emporschrauben des Steuertarifs gefährdete die wirtschaftliche Existenz und die persönliche Freiheit der Untertanen. Dieses Versagen des Absolutismus rief eine Gegenbewegung in fast allen Ländern und eine neuartige steuerpolitische Idee hervor. Diese Idee war das Ergebnis eines neuen wissenschaftlichen Bewusstseins; denn die Menschen waren trotz Humanismus und Reformation abgestoßen von den Spaltungen, die das geistige, politische und religiöse Leben durchzogen. In dieser neuen Zeit erstrebten die Menschen einen sicheren Halt und einige Vordenker glaubten, diesen an den der Naturordnung entsprechenden unveränderlichen Begriffen und Normen zu finden. ${ }^{251}$

Da auch in der Politik die Idee der Staatsräson den Menschen keinen festen Beistand hatte bieten können, trat die Frage nach Gerechtigkeit verstärkt in den

247 Schwennicke, Andreas: „Ohne Steuer kein Staat“, S. 353.

248 Die Unterscheidung zwischen direkten und indirekten Steuern geht auf den französischen Physiokraten Quesnay zurück; vgl. Gömmel, Rainer/Klump, Rainer: Merkantilisten und Physiokraten in Frankreich. Geschichte der volkswirtschaftlichen Lehrmeinungen, Darmstadt 1994, S. 133 f. Da diese Unterteilung nach wie vor geläufig ist und in den zeitgenössischen Diskussionen ständig vorgenommen wurde, wird sie hier beibehalten.

249 Mann, Fritz Karl: Steuerpolitische Ideale, S. 99.

250 Ebd., S. $80 \mathrm{ff}$.

251 Ebd., S. 81. 
Vordergrund. ${ }^{252}$ Was aber macht eine gerechte Ordnung aus, woran kann man eine ungerechte Ordnung erkennen? Was sind die Kriterien der Gerechtigkeit, und wie kann man Gewissheit über sie gelangen? Gibt es bestimmte Regeln oder Prinzipien, die gleichsam aus sich heraus eine gerechte Ordnung oder eine gerechte Entscheidung hervorbringen? ${ }^{253}$

Ein naheliegender Antwortversuch besteht darin, sich bestimmter Regeln und Handlungsweisen zu bedienen, die gleichsam aus sich heraus eine gerechte Ordnung hervorbringen. Die bekannteste Regel hat Eingang in das römische Recht gefunden: „Iustitia est constans et perpetua voluntas ius suum cuique tribuendi“ (Die Gerechtigkeit ist der beständige und dauerhafte Wille, jedem sein Recht zukommen zu lassen), wie es der römische Jurist Ulpianus formulierte. Die Formel „jedem das Seine" (suum cuique) ist jedoch inhaltsleer, da sie letztlich zur Rechtfertigung jeder beliebigen Ordnung dienen kann. ${ }^{254}$

Womöglich könnte die Rechts- und Staatsphilosophie die Antwort auf die Frage nach der gerechten sozialen und staatlichen Ordnung geben. In der Antike wurde gefragt: Welche Herrschaft ist gerecht? In der Neuzeit hingegen: Ist Herrschaft überhaupt gerecht?

Für die rationalistische Naturrechtslehre des 17. und 18. Jahrhunderts mit ihren bedeutendsten Vertretern wie Thomas Hobbes, John Locke, Jean-Jacques Rousseau und Immanuel Kant war der Staat ein im Grunde künstliches Produkt, und der Mensch wurde zum Herrscher und Beherrscher seiner selbst, zum „autonomen Produzenten seiner Geschichtswelt“ ${ }^{255}$ Vor allem dachte die Naturrechtslehre des 17./18. Jahrhunderts entschieden vom Individuum her, was man deutlich an der Verwendung der beiden tragenden Hauptelemente der neuen Lehre sieht: dem Gedanken des Naturzustandes und der Lehre vom Gesellschafts- beziehungsweise Herrschaftsvertrag. Da im Naturzustand alle Menschen frei und gleich sind, gründet sich die Herrschaft auf der Zustimmung der Herrschaftsunterworfenen. Dieser Gedanke „ist bis auf den heutigen Tag die entscheidende Legitimationsfigur staatlicher Ordnung und Herrschaft. " ${ }^{256}$

Zur Legitimation der Herrschaft trat ihre Limitation. Bei Hobbes gab es nur den souveränen Staat, bei Locke - den man den Begründer des Liberalismus nennt ${ }^{257}$ -

252 Ebd., S. 82.

253 Dreier, Horst: „Wir hatten Gerechtigkeit erhofft ... doch bekommen haben wir den Rechtsstaat", in: Frankfurter Allgemeine Zeitung vom 05.01.2015, S. 6.

254 Ebd., S. 6.

255 Ebd., S. 6

256 Ebd., S. 6.

257 Ebd., S. 6; Locke, John: www.Philosophenlexikon.de (letzter Zugriff 20.02.2017). 
erweiterte sich dieser zum Rechtsstaat. Jean-Jacques Rousseaus Gesellschaftsvertrag („Du Contrat Social ou Principes du Droit Politique“, 1762) ist die Antwort auf die Frage, wie individuelle Freiheit und staatliche Ordnung miteinander vereinbar sind. Ihm zufolge lag die Funktion der Vorstellung eines ursprünglichen Vertrages darin, jeden Gesetzgeber darauf zu verpflichten, seine Gesetze so zu erlassen,

als ob sie aus dem vereinigten Willen eines ganzen Volkes haben entspringen können, und jeden Untertan, sofern er Bürger sein will, so zu sehen, als ob er zu einem solchen Willen mit zusammengestimmt habe. Denn das ist der Probierstein der Rechtmäßigkeit eines jeden öffentlichen Gesetzes. ${ }^{258}$

Kant meinte in seiner Rede von der „wahren Republik“ im Kern eine repräsentative Demokratie, flankiert von rechtsstaatlichen Garantien und dem zentralen Recht eines jeden auf Freiheit. ${ }^{259}$

Wie konnte ein „natürliches“ System der Besteuerung gefunden werden? Den Ansatz hierfür bildete der politische und wirtschaftliche Individualismus, den insbesondere Locke mit dem Naturrecht verband. Man glaubte, dass - wie in der Natur - auch in der Wirtschaft Freiheit herrschen müsse und jeder staatliche Eingriff schädlich wäre. Das Neuartige dieser Gedanken war, dass die Freiheit in der Wirtschaft als ein für alle ökonomischen Vorgänge verbindliches Naturgesetz verkündet wurde. ${ }^{260}$

Schritt für Schritt drangen - neben den Idealen des Absolutismus - die naturrechtlichen Gedanken in die Regierungskreise und die politische Opposition vor, sie wurden zum wissenschaftlichen und politischen Rüstzeug der kommenden Elite, bis diese die politische Herrschaft übernahm. ${ }^{261}$

\subsubsection{Die Ansätze einer Steuerrechtslehre als organische Einheit}

Sehr fruchtbar war der Versuch, den Blutkreislaufgedanken auf das Gebiet der Finanzwirtschaft zu übertragen. Wenn Zufluss und Abfluss öffentlicher Gelder als eine organische Einheit begriffen wurden, dann stellte die Steuer keinen lästigen Fremdkörper mehr dar, sondern war ein Teil eines natürlichen Verlaufs. Damit

258 Rousseau, Jean-Jacques, zitiert bei: Dreier, Horst: „Wir hatten Gerechtigkeit erhofft“, S. 6

259 Kant, Immanuel, zitiert bei: Dreier, Horst: „Wir hatten Gerechtigkeit erhofft“, S. 6

260 Mann, Fritz Karl: Steuerpolitische Ideale, S. 85.

261 Ebd., S. 87. 
war eine der praktischen Finanzpolitik einleuchtende Rechtfertigung der Steuer gefunden. ${ }^{262}$

Der französische Philosoph François de la Mothe le Vayer (1588-1672) hat für diese im 17. Jahrhundert entstandene Lehre einen anschaulichen Naturvergleich angeführt: Die Mehrzahl der Steuern sollte den Dämpfen gleichen, die von der Erde aufsteigen und sich zu Wolken verdichteten, dann niedersanken und an den Ort zurückkehrten, von dem sie ausgegangen sind. ${ }^{263}$ Bei ihm sucht man vergeblich nach ethischen Normen; sein Prinzip wurde nicht aus der Gerechtigkeitsidee hergeleitet, sondern Ausgangspunkt und Ziel seiner Betrachtung war der Fortbestand des Staates. Die Staatserhaltung jedoch setzte den Fortbestand der Finanzwirtschaft voraus. ${ }^{264}$

De la Mothe le Vayer rechtfertigte die Gleichmäßigkeit der Lastenverteilung mit dem Staatsinteresse und strebte eine Lastenverteilung an, die sich nach den wirtschaftlichen Kräften richtete. Das wäre nur möglich, wenn die Fähigkeit der Bürger zur Steuerzahlung erhalten bliebe und die Steuerlast der Leistungsfähigkeit der Bürger angepasst würde. Darüber hinaus dürfte es grundsätzlich keine Steuerbefreiung geben. Damit hat François de la Mothe le Vayer im 17. Jahrhundert im Kampf gegen die Steuerprivilegien der oberen Stände ein eindrucksvolles wirtschaftspolitisches Argument formuliert, das in die naturrechtliche Steuerlehre einging und bis in die heutige Zeit Bestand hat. ${ }^{265}$

\subsubsection{Das Gerechtigkeitsideal im Zeitalter der Aufklärung}

\subsubsection{Die Auffassungen über Steuergerechtigkeit}

Die Mehrzahl der Autoren, die auf dem Boden des Naturrechts standen, wollten nicht das überkommene Gefüge der Besitzverteilung verändern, sondern Belastungskorrekturen im Wege einer Steuerreform durchführen. Nur dieses Ziel war mit den Forderungen nach Allgemeinheit und Gleichmäßigkeit der Besteuerung gemeint. Hauptsächlich im 18. Jahrhundert verbreitete sich die Ansicht, dass die Besteuerung lediglich die Wirtschaft begleiten sollte, ohne diese zu stören. Auch aus diesem Grunde sollte die Steuer keine „außerfiskalischen“ Zwecke erfüllen;

262 Ebd., S. 88; vgl. die weiteren Ausführungen in Kapitel 5.3.3.5.

263 Mann, Fritz Karl: Steuerpolitische Ideale, S. 90.

264 Ebd., S. 94.

265 Ebd., S. 95. 
diese Auffassung erschien zur damaligen Zeit nicht nur als zweckmäßig, sondern insbesondere auch als gerecht. ${ }^{266}$

Es waren die großen politischen Autoren der Aufklärungszeit, die mit den Vorschlägen der Steuerprogression und der Freilassung des Existenzminimums die öffentliche Meinung für die beiden sozialpolitischen Maßnahmen gewannen. ${ }^{267}$ Im 17. Jahrhundert vertrat Kardinal Richelieu (1585-1642) den Grundsatz, die Herrscher sollten sich „den Überfluss der Reichen zunutze machen, bevor sie die Armen durch außerordentliche Lasten anstrengen“.268 Zur gleichen Zeit rechtfertigte Kaspar Klock (1583-1655) die Steuerfreiheit der Armen damit, dass nur die Früchte durch die Steuer erfasst werden dürften und diese daher beim Mangel eines Vermögens oder eines Ertrages fortfallen müssten. ${ }^{269}$

Ein Jahrhundert später griff Montesquieu (1689-1755) den Progressionsgedanken auf, doch er empfahl stattdessen einen degressiven Tarif für die Personalbesteuerung. Unverbindlicher setzte er sich für die Freihaltung des physischen Existenzminimums ein, und zwar sowohl bei der Personal- als auch bei der Grundsteuer. Mit Montesquieu begann die Bildung eines neuen naturrechtlichen Gleichheitsideals, die Vorschläge der Steuerdegression und der Freilassung des Existenzminimums konnten sich sowohl aus dem Naturrechtsideal als auch aus dem Gerechtigkeitsideal voll entfalten. ${ }^{270}$

Jean-Jacques Rousseau (1712-1778) ging von der allgemeinen Auffassung aus, dass die Steuer nach der Größe des Vermögens abgestuft werden müsste, doch bedurfte nach seiner Ansicht die Proportionalität einer erheblichen Korrektur. Rousseau schlug deshalb einen radikalen Weg ein, indem er darauf hinwies, dass das physische Existenzminimum bei allen Menschen gleich wäre, deshalb müsste das Lebensnotwendige steuerfrei bleiben; das Überflüssige könnte im Notfall ganz weggesteuert werden. Schien bisher die Progression als Ausfluss der Steuergerechtigkeit ein unentbehrliches Mittel einer Belastung nach den Fähigkeiten zu sein, so wurde nun der soziale Aspekt in den Mittelpunkt der Auseinandersetzung gerückt.

266 Petty, William: A Treatise of Taxes and Contributions, London 1662, in: Hull, Charles Henry (Hrsg.): The Economic Writings of Sir William Petty, Cambridge 1899, S. 1-98, S.32.

267 Mann, Fritz Karl: Steuerpolitische Ideale, S. 160.

268 Richelieu, Armand-Jean du Plessis, Premier Duc: Politisches Testament und kleinere Schriften (1633), übers. von Frieda Schmidt (Klassiker der Politik 14), Bremen 1984, S. 120.

269 Klock, Caspar: De contributionibus hodie ut plurimum in Germania usitatis. Dissertatio Theoretico-practica., Basel 1608, S. 58, zitiert bei: Mann, Fritz Karl: Steuerpolitische Ideale, S. 160.

270 Mann, Fritz Karl: Steuerpolitische Ideale, S. 160 f. 


\subsubsection{Die ethischen Steuertheorien}

Um sich vor der Willkür und den Übergriffen des absoluten Fürstenstaates des 17. und 18. Jahrhunderts zu schützen, stellte sich die Frage, wo die natürliche Grenze der Besteuerung lag. Der politische, soziale und ökonomische Individualismus, der sich mit dem vordringenden Naturrecht verband, zielte auf die Schwächung des Staates ab; er wollte zwischen der Privatsphäre und der Staatsphäre eine feste Grenze ziehen. Es musste ein Prüfstein gefunden werden, um die gerechte von der ungerechten Steuer zu trennen. Damit trat die Idee der Steuergerechtigkeit in den Mittelpunkt der wissenschaftlichen Diskussionen, die ethische Steuertheorien befruchteten. ${ }^{271}$

Der englische Autor Thomas Hobbes hat die Steuer als einen Preis für den Frieden bzw. als Lohn für die Verteidigung der Bürger beschrieben. ${ }^{272}$ Der Staatsrechtslehrer Samuel von Pufendorf (1632-1694) gilt als der erste deutsche Autor, der die Steuern grundsätzlich als ein Entgelt für den dem Einzelnen für Leben und Vermögen gewährten Schutz betrachtete. Auf dieser Assekuranztheorie baute von Pufendorf eine Kombination von Kopfsteuern für den Schutz des Lebens und von Steuern, die nach der Größe der aus dem Vermögen gezogenen Einkünfte bemessen werden sollen, auf. Eine Ergänzung sollte diese direkte Besteuerung durch Konsumtionssteuern finden, deren Bedeutung nach der Not durch den Dreißigjährigen Krieg in der Theorie lange Zeit nicht mehr gewürdigt worden war. ${ }^{273}$

In diesen Ansichten, die bei verschiedenen Autoren in ähnlicher Form dargelegt wurden, lag eine entscheidende Wende im steuerpolitischen Denken. Aus der Idee der Gerechtigkeit wurden sowohl die Notwendigkeit der Besteuerung als auch die Steuerhöhe sowie die Steuerverteilung abgeleitet. ${ }^{274}$

Während der Absolutismus eine steuerpolitische Harmonie von Fürst und Volk vorausgesetzt hatte, wurden nunmehr der Staat und die Bürger als gleichberechtigte Partner auf steuerlichem Gebiet anerkannt. ${ }^{275}$ Die Assekuranztheorie als Versicherungsprämie für den staatlichen Schutz von Leben und Eigentum formte die öffentliche Meinung in Steuerfragen. Die beiden Grundsätze der Besteuerung,

271 Ebd., S. 104.

272 „The wages due to them that hold the publique Sword, to defend private men in the exercise of several Trades and Callings." Hobbes, Thomas: Leviathan. The Matter, Form and Power of a Commonwealth Ecclesiastical and Civil (1658), London 1886; auf: https: ebooks/adelaide.edu.au/h/hobbes/Thomas /h681/ chapter 30.html (letzter Zugriff: 28.02.2017).

273 Wilke, Gustav: Die Entwicklung der Theorie, S. 9.

274 Mann, Fritz Karl: Steuerpolitische Ideale, S. 107.

275 Ebd., S. 109 f. 
das Allgemeinheits- und das Gleichmäßigkeitspostulat, wurden zu politischen Glaubensartikeln. ${ }^{276}$ Die Erfolge, die der Individualismus und das Naturrecht im 17. Jahrhundert erzielten, haben auch zur Verbreitung der assekuranztheoretischen Grundansicht beigetragen, denn diese entsprach zugleich den innerpolitischen Zielen: der Brechung der Standesherrschaft. ${ }^{277}$

Die naturrechtlichen Steuerpostulate waren Spiegelbild der politischen Lage und können daher als Gradmesser des Freiheitsdranges und der Demokratisierung dienen. Diese fanden in der Erklärung der Menschen- und Bürgerrechte vom 26. August 1789 in Frankreich - sowie später in vielen Verfassungen - ihren Niederschlag.

\subsubsection{Das Ideal eines rationalen Steuersystems}

Zu Beginn des Jahres 1707 publizierte Sébastien Le Prestre de Vauban (1633-1707) - der eine Karriere im Dienst des Königs Ludwig XIV. gemacht hatte - anonym die Schrift „Projet d'une dîme royale“ („Projekt eines königlichen Zehnten“), in welcher er die Steuerpolitik des französischen Absolutismus im Hinblick auf die unterdrückten Schichten kritisierte. Er schlug eine Reform der Steuern vor und verteilte die Schrift an zahlreiche Freunde und politisch interessierte Bekannte. Vauban trug das Projekt dem König vor, es wurde jedoch abgelehnt, da es mit seiner gleichmäßigen Besteuerung aller Abgaben in Höhe eines Zehntels auch den bis dahin weitgehend steuerfreien Adel belastet hätte. Die Schrift wurde gleich nach dem Erscheinen verboten. ${ }^{278}$

Obwohl es sich um ein praktisches Problem, Gesundung der französischen Staatsfinanzen, handelte, entwickelte Vauban rationale Grundsätze als Fundament einer natürlichen Finanzverfassung. Seine „Grundmaximen“, die „Maximes fondamentales de ce Système “279, enthielten eine assekuranztheoretische Begründungs- und Verteilungslehre: Die Untertanen benötigen den staatlichen Schutz, und der Souverän des Staates das Geld der Untertanen, um ihnen diesen Schutz zu gewähren. Da der Staatsbedarf von der Hilfe der Untertanen abhängt, müssen auch alle Untertanen dazu beitragen. Vauban strebte in erster Linie eine gleichmäßige Lastenverteilung an, ohne dass sich jemand dieser Verpflichtung entziehen können sollte. Anpassung an die Leistungsfähigkeit und Gleichmäßigkeit der Belastung bedeuteten für Vauban nichts anderes als die Wiederherstellung der Naturordnung. ${ }^{280}$

276 Ebd., S. 110.

277 Ebd., S. 111.

278 Ebd., S. 117.

279 Vauban, Sébastien Le Prestre: Dîme Royale (1707), zitiert in Mann, Fritz Karl: Steuerpolitische Ideale, S. 117.

280 Mann, Fritz Karl: Steuerpolitische Ideale, S. 118 f. 
Um zu verstehen, aus welcher Situation heraus Vauban die Steuerpolitik des französischen Absolutismus kritisierte, müssen wir auf die staatspolitische Situation der damaligen Zeit zurückblicken:

Im 17. Jahrhundert ging mit der Festigung der Zentralgewalt eine stete Zunahme der finanziellen Aufwendungen einher, die sich aus den Repräsentationsansprüchen des Hofes und des sich bei Hofe versammelnden hohen und niederen Adels und der Geistlichkeit ergaben. Die Ausbildung des zentralisierten Staatswesens und die Wahrung seiner inneren und äußeren Interessen bedingten gleichfalls erhebliche Finanzmittel. ${ }^{281}$ Ebenso war es erforderlich, dem Königreich mit militärischen oder diplomatischen Mitteln den gebührenden internationalen Rang zu sichern und ihn notfalls zu verteidigen. ${ }^{282}$

Die Finanzquellen, aus denen diese erheblichen Aufwendungen des absolutistischen Systems gespeist wurden, waren längst nicht ausreichend. Aus dem Widerspruch zwischen den stetig wachsenden finanziellen Forderungen des Königs, des Hofes, des Adels und des Staatsapparates, insbesondere des Militärs, einerseits und den begrenzten Möglichkeiten, die das überkommene Steuer- und Abgabensystem boten, andererseits, ergab sich für Jean-Baptiste Colbert (1619-1683) die Notwendigkeit, einen ungewöhnlichen Weg einzuschlagen. Seine staatlich gelenkte Förderung des Handels hatte zweierlei zur Folge: Zum einen erhöhte sie den finanziellen Spielraum für die Herrschenden, denn es gelang Colbert bis 1672, das Budget auszugleichen. ${ }^{283}$ Zum anderen erhielt der Modernisierungsprozess im Königreich Frankreich durch die wesentlichen Veränderungen in der Sozialstruktur einen ungewöhnlich starken Impuls.

Die finanziellen Leistungen, die das Finanzwesen hervorgebracht hat, haben Frankreich von 1500 bis 1789 zur führenden Macht Europas gemacht. Diese Finanzen schufen den ersten national monarchischen Einheitsstaat Europas, jedoch zu einem hohen Preis: ein Teil der Bevölkerung stand vor dem Hungertod, das Land vor der Revolution; ${ }^{284}$ denn die direkte Hauptsteuer, die „Taille“, traf vornehmlich die ländliche bäuerliche und abhängige Bevölkerung. Außer der Taille wurde im 18. Jahrhundert zudem die "Capitation“, eine Art allgemeine klassifizierte Standes-, Berufs-, Personal- und Einkommensteuer eingeführt. Dazu kamen der „Zehnte“ und "Zwanzigste“, die in der Zeit des Spanischen Erbfolgekriegs 1710 eingeführt wurden. Der damalige „Zehnte“ war eine allgemeine Einkommensteuer aller

281 Birjukowitsch, W. W. u. a.: Geschichte der Neuzeit, 1. Bd., Berlin 1954, S. 185-187.

282 Markov, Walter u.a. (Hrsg.): Kleine Enzyklopädie Weltgeschichte, Leipzig 1964, S. 224.

283 Herzfeld, Hans (Hrsg.): Geschichte in Gestalten, S. 267.

284 Schmoller, Gustav von: Skizze einer Finanzgeschichte, S. 12 f. 
Art von Einkommen. ${ }^{285}$ Besonders drückend für die sogenannten kleinen Leute war die in Stadt und Land auf Salz erhobene indirekte Steuer, die "Gabelle“, die bereits 1341 eingeführt und im Jahr 1790 wieder abgeschafft wurde. Neben den Geldleistungen bestanden sehr schwere Naturallasten, und die Steuerverteilung war die denkbar ungerechteste, wie Schmoller ausführt. ${ }^{286}$ Das Erdrückendste für Volk und Finanzen aber war die Form der Erhebung durch die Steuerpächter, eine bis ins 18. Jahrhundert hinein vorherrschende Form der Privatisierung des Steuereintreibens. Die Regierung war in ihren Händen, weil sie auf Jahre hinaus Vorschüsse von den Steuerpächtern erhielten und diese mit staatlicher Autorität ausgestattet rigoros gegen die Untergebenen vorgehen konnten. Sie erhielten in der Regel 25 Prozent der Steuersumme und 15 Prozent für die Vorausbezahlung und trieben außerdem vom Volk ein Mehrfaches der Steuerschuld ein. ${ }^{287}$

Die Regierenden sahen die enormen Einnahmen als etwas an, das sie wie Privatleute als ihr eigenes Vermögen benutzen durften. Sie scheuten vor dem ungerechtesten Steuerdruck nicht zurück, weil sie die Folgen damals nicht überblickten. ${ }^{288}$ Für sie waren die "Maximes fondamentales"von Vauban - der in erster Linie eine gleichmäßige Lastenverteilung anstrebte - etwas völlig Fremdes und Unverständliches.

Obwohl die Schrift von Vauban sachliche Mängel enthielt - so unterschied dieser u. a. nicht Rohertrag und Reinertrag -, schuf dieser doch mit seiner „Dîme royale" die Idee eines Systems der Staatseinnahmen. ${ }^{289}$ Mit der Verankerung des Allgemeinheits- und Gleichmäßigkeitspostulates in einem rationalistischen Steuersystem wurde durch Vauban die Möglichkeit einer Verbreitung dieser beiden Forderungen über die ganze Welt ermöglicht. Wir treffen sie in allen maßgeblichen Schriften des Aufklärungszeitalters und finden sie in allen Verfassungen bis in unsere jüngste Gegenwart. ${ }^{290}$

\subsubsection{Die direkte Besteuerung als Ideal}

Die neue geistige Haltung strahlte auf alle Bereiche der Steuerpolitik aus. Überall zeigten sich Ansätze zu einem Bündnis der Ökonomie mit der mächtigsten geistigen Bewegung dieser Zeit, dem revolutionären, individualisierten Naturrecht. Der Glaube an die Naturordnung führte allmählich zu einem völligen Meinungs-

285 Wagner, Adolph: Finanzwissenschaft 3. Teil, S. 161.

286 Schmoller, Gustav von: Skizze einer Finanzgeschichte, S. 15.

287 Ebd., S. 15.

288 Ebd., S. 16.

289 Mann, Fritz Karl: Steuerpolitische Ideale, S. 121.

290 Ebd., S. 123. 
umschwung. ${ }^{291}$ Die Vorzüge der Akzise waren überwiegend mit ihrer fiskalischen Ergiebigkeit, der Einfachheit der Erhebung, dem Antrieb zur Sparsamkeit sowie mit ihrer Unmerklichkeit begründet worden. Die Frage der Gerechtigkeit trat hinter diesen praktischen Erwägungen zurück.

Das Naturrecht hatte dagegen jedoch ein neues Ziel gesetzt: Das Haupterfordernis der Besteuerung war die Gerechtigkeit!292 Um diese zu erreichen, mussten - unter Berücksichtigung der in der öffentlichen Meinung anerkannten Assekuranztheorie - Staatsleistung und Steuerleistung verglichen werden. Nur diejenige Steuer war gerecht, deren Höhe den durch die Staatstätigkeit erzielten individuellen Vorteilen oder zumindest dem anteiligen Kostenanteil daran entsprach. Dabei konnte vorausgesetzt werden, dass der Reiche aus dem staatlichen Schutz mehr Vorteile zog als der Arme. Dies führte zu der Überzeugung, dass man als Maßstab für die Steuerhöhe an das Vermögen, den Ertrag und das Einkommen anknüpfen könne. Damit führten die vom Naturrecht ausgehenden Überlegungen zwangsläufig zu einer Bevorzugung der direkten Steuern. ${ }^{293}$

Langsam vollzog sich der Meinungsumschwung in den meisten Ländern Europas. Voran ging Frankreich, das nicht im gleichen Umfang wie Deutschland, Holland und England das Akziseideal umgesetzt hatte. Reformpolitiker plädierten in der Mehrzahl für eine Aufhebung der indirekten Steuern oder wenigstens für die Umwandlung der indirekten in direkte Steuern.

Nach dem Tode Ludwigs XIV. wandte sich die neue Regierung unter Philipp d'Orléans an die breite Öffentlichkeit und lud diese zur Mitarbeit ein. Die Öffentlichkeit reagierte mit zahlreichen Plänen, um die Steuern zu erleichtern, die Staatslasten zu vermindern und den Handel zu heben.

Die zur finanziellen Sanierung des absolutistischen Frankreichs entwickelten Pläne entsprangen einem tief verwurzelten naturrechtlichen Denken: Die willkürliche und ungerechte Steuerverfassung der Vergangenheit sollte durch ein auf ethischer Grundlage errichtetes Steuersystem abgelöst werden. Entsprechend der vom Naturrecht verlangten Allgemeinheit der Steuerpflicht sprach sich eine überwältigende Mehrheit der Autoren für die direkte Besteuerung aus. ${ }^{294}$

Dieses Gedankengut schlug sich auch in Deutschland, Österreich, Italien und England nieder. Karl Ferdinand Pescherinus griff auf das neue Finanzsystem von Vauban zurück und ließ in seiner Nachahmung der „Dîme royale“ zwei Steuer-

291 Ebd., S. 124.

292 Ebd., S. 124.

293 Ebd., S. 125; nach der älteren Ausdrucksweise war nur diejenige Steuer gerecht, die unmittelbar nach den „Fähigkeiten“ abgestuft war.

294 Ebd., S. 133. 
gruppen fortbestehen: die „Generalzehenden“ und die Ein- und Ausfuhrzölle. Alle anderen Verkehrs-, Verbrauch- und Luxussteuern sowie das Salzmonopol entfernte er aus dem Vauban'schen Fonds. ${ }^{295}$

Obwohl die meisten Vorschläge einer direkten Steuer verbunden waren mit einer schroffen Ablehnung der indirekten Abgaben, waren viele wissenschaftliche und politische Schriftsteller nicht bereit, dieser Zeitströmung zu folgen. Sie vertraten den Standpunkt, dass direkte Steuern weit davon entfernt wären, gerecht zu sein; denn diese seien die willkürlichsten Zwangsabgaben. Zu verstehen sind diese Auffassungen daraus, dass die Finanztechnik zu Beginn des 18. Jahrhunderts zu rückständig war, um der durch das Naturrecht verlangten Allgemeinheit und Gleichmäßigkeit der Besteuerung und damit der Gerechtigkeit zu genügen.

In Kriegszeiten jedoch waren die allgemeinen direkten Steuern Wegbereiter für die erfolgreiche Finanzierung der Kriegslasten. Ludwig XIV. hat mit einer allgemeinen klassifizierten Kopfsteuer im Orléanischen Krieg (1695) sowie durch den allgemeinen Zehnten im Spanischen Erbfolgekrieg (1710) die Ständeprivilegien durchbrochen. Der englische Premierminister William Pitt hat 1799 eine allgemeine Einkommensteuer für die Dauer des Krieges gegen Frankreich eingeführt. Diese neue Einkommensteuer trug maßgebend dazu bei, dass Napoleon besiegt werden konnte, weshalb diese einmal später als die Steuer bezeichnet wurde, die Napoleon bezwang. ${ }^{296}$ Obwohl sowohl im wissenschaftlichen und politischen Schrifttum als auch in der vom Kriegsbedarf ausgehenden Entwicklung mehr und mehr den direkten Steuern Vorrang eingeräumt wurde, blieben die europäischen Steuerverfassungen noch lange unter der Vorherrschaft der indirekten Abgaben. ${ }^{297}$

\subsubsection{Die Vision einer Alleinsteuer}

In der kameralistischen Auseinandersetzung mit der Vision einer einzigen Steuer begegneten sich zwei völlig verschieden gelagerte wissenschaftliche Richtungen. Einerseits strebte die deutsche Kameralistik nach einem praktischen, einfachen und gerechten Steuersystem; denn grundsätzlich bestand Einigkeit darüber, dass

295 Mann, Fritz Karl: Der Marschall Vauban und die Volkswirtschaftslehre des Absolutismus. Eine Kritik des Merkantilsystems, München/Leipzig 1914, S. 469 ff.

296 Bundesministerium der Finanzen: Neue Ideen und Wege der Besteuerung im 18. und frühen 19. Jahrhundert, vom 09.12.2010, auf: http://www.bundesfinanzministerium. de/Content/DE/Standardartikel/Ministerium/Geschaeftsbereich/Bundesfinanzakademie/Steuermuseum/Museumsfuehrer/10-neue-ideen-und-wege-der-besteuerung. html (letzter Zugriff: 20.02.2017), S. 2.

297 Mann, Fritz Karl: Steuerpolitische Ideale, S. 143. 
weniger Steuerarten besser sind als mehr Steuerarten. Andererseits lehnte man die Ideen des Physiokratismus ab. ${ }^{298}$

Die ersten einflussreichen Alleinsteuerpläne wurden von den Lehren des Naturrechts des 17. Jahrhunderts geprägt. Mann unterscheidet drei Gruppen: ${ }^{299}$

Nach Auffassung der ersten Gruppe sollten unabhängig von Steuergegenstand, Steuermaßstab und Steuertarif alle Steuern letzten Endes von den Grundeigentümern getragen werden, da alle Gewinne auf der ausschließlichen Produktivität des Bodens beruhten. John Locke hat in seiner Schrift über die Zinssenkung und die Geldwertsteigerung diesen Gedanken aufgegriffen und aus ihnen das Programm einer einzigen Grundsteuer abgeleitet. Schon die Persönlichkeit des Verfassers sicherte der Schrift eine Resonanz.

Zur zweiten Gruppe gehören mehrere französische Pläne vom Beginn des 18. Jahrhunderts. Das Allheilmittel sollte eine Grundsteuer sein, deren Erhebung nicht den staatlichen Beamten, sondern Pächtern und Unterpächtern anvertraut werden sollte. Die Vorschläge gingen jedoch weit auseinander; so sollte die einzige Steuer sowohl das Vermögen als auch das Einkommen erfassen, in anderen Fällen wurde sie auf das Vermögen beschränkt. Ebenfalls wurde eine einzige Klassensteuer, eine einzige Grundsteuer oder auch eine Haussteuer empfohlen, die ergiebig genug wäre, die meisten Zwangsabgaben zu ersetzen. So sollten weiterhin die inneren Verkehrsschranken und Binnenzölle und die Privilegien der oberen Stände beseitigt werden. Fernerhin sollten sogar Wohlhabende und Reiche grundsätzlich steuerfrei sein, wenn sie kein Grundeigentum besaßen: Das waren Beamte, freie Berufe, die Gewerbetreibenden und die Kaufleute. In den fortschrittlicheren Ländern, in England und Frankreich, reihte sich Versuch an Versuch, den Plan der Alleinsteuer heimisch zu machen. Doch der Widerspruch zu den ethischen Grundsätzen war zu offensichtlich, die Diskussionen fanden nur im akademischen Bereich ihren Widerhall und kamen der Verwirklichung nicht näher.

Zur dritten Gruppe zählten die Physiokraten, die aus wenigen Obersätzen ein vollständiges Regelwerk des politischen, gesellschaftlichen und wirtschaftlichen Lebens abgeleitet haben. Der Gründer der Physiokratie, François Quesnay (1694-1774), wollte ähnlich den Philosophen des griechischen Altertums ein umfassendes Wissenschaftssystem begründen, das Ökonomie mit Politik, Sozial-ethik und anderen Lehren des Gesellschaftslebens verband. Wenn es eines Beweises für

298 Jenetzky, Johannes: System und Entwicklung, S. $133 \mathrm{f}$.

299 Mann, Fritz Karl: Steuerpolitische Ideale, S. 168-201. 
die zeitgemäße Verbindung von naturrechtlicher Philosophie und ökonomischer Analyse bedürfte, so wäre die Entstehung der Physiokratie ein schlagender Beleg. ${ }^{300}$

Innerhalb der physiokratischen Wirtschaftslehre gingen die stärksten Anregungen von der organizistischen Gesamtbetrachtung aus. ${ }^{301}$ Die Hauptleistung dieser bereits ein Jahrhundert vor Quesnay entwickelten Finanzlehre war der Versuch, den Kreislaufgedanken auf die Finanzwirtschaft zu übertragen. Der Arzt und Ökonom Quesnay wendete die Erkenntnis des Blutkreislaufs auf die „économie politiqu“ an. Zwar blieb der unmittelbare Einfluß Quesnays national beschränkt, ${ }^{302}$ doch Joseph Schumpeter hat seine Würdigung für Quesnay wie folgt zum Ausdruck gebracht:

\begin{abstract}
All essential ideas and at the same time all the vigour of an outstanding personality were combined in François Quesnay, who had created these ideas from within himself to a far greater degree than any other economist had done. He was one of the greatest and most original thinkers in our field. ${ }^{303}$
\end{abstract}

Die physiokratischen Gedankengänge bewegten sich auf einer ungleich höheren Ebene als sämtliche Alleinsteuerpläne der Vergangenheit. Quesnay unterschied drei Gesellschaftsklassen: ${ }^{304}$ Zur landwirtschaftlichen Bevölkerung gehörten die beiden ersten Klassen, der produktive Ackerbauer und der nicht unmittelbar produktive Grundeigentümer. In der dritten Klasse versammelten sich alle Übrigen, insbesondere die Gewerbetreibenden und Händler, die nur dadurch leben konnten, dass sie von den beiden anderen Klassen unterhalten wurden.

Da der Wirtschaftstheorie der Physiokraten zufolge ausschließlich die Landwirtschaft eine Nettowertschöpfung („produit net“) hervorbringen kann, sollten alle Steuern aus diesem Überschuss finanziert werden. Es wäre deshalb auch nur diejenige Steuer gerecht, die den "produit net“ erfasst; denn alle Steuern, die auf andere Erträge als den Bodenertrag erhoben würden, stellten nur einen Umweg dar und würden daher auf die produktiven Klassen überwälzt werden. Dieser Umweg - so wurde argumentiert - erhöhte die Erhebungskosten, konnte aber nicht verhindern, dass im Endergebnis nur der „produit net“ erfasst würde. ${ }^{305}$

300 Salin, Edgar: Geschichte der Volkswirtschaftslehre, in: Spiethoff, Arthur (Hrsg.): Enzyklopädie der Rechts- und Staatswissenschaft, Abteilung Staatswissenschaft, Berlin/ Heidelberg 1923, S. 1-44, S. 14.

301 Mann, Fritz Karl: Steuerpolitische Ideale, S. 187.

302 Salin, Edgar: Geschichte der Volkswirtschaftslehre, S. 17.

303 Schumpeter, Joseph A.: Economic Doctrine and Method. An Historical Sketch, London 1957, S. 44.

304 Mann, Fritz Karl: Steuerpolitische Ideale, S. 188 ff.

305 Ebd., S. 190. 
In Frankreich fanden die physiokratischen Gedanken sowohl in der verfassungsgebenden als auch in der gesetzgebenden Nationalversammlung nach dem 9. Juli 1789 Beachtung. Die neuen Machthaber wagten es jedoch nicht, das Schicksal des Staates von einer einzigen Steuer abhängig zu machen; von Anfang an wurde an eine Mehrheit von Staatssteuern gedacht, im Bericht von François de La Rochefoucauld vom 18. August 1790 fand sich sogar ein weit verästeltes Steuersystem. ${ }^{306}$

Ein physiokratisches Experiment wurde in Deutschland durch den reformeifrigen Markgrafen Karl Friedrich von Baden-Durlach (1728-1811) gewagt, der an Stelle aller früheren Abgaben eine einzige Grundsteuer einführte. Heftige Beschwerden der Bevölkerung setzten jedoch ein, da der Reinertrag der Grundstücke nicht zu ermitteln war und dieser zu hoch geschätzt wurde. Das neue Verfahren wurde bald wieder eingestellt, in der Gemeinde Dietlingen hielt es sich jedoch bis zum Jahr $1802 .{ }^{307}$

Ansatzpunkt der antiphysiokratischen Kritik war die Lehre vom Boden als alleiniger Quelle aller Reichtümer. In der Literatur wurde bald dargelegt, dass alle arbeitenden Menschen den Wert der produzierten Gegenstände erhöhten und letztendlich jeder Gewinn auf dem Zusammenwirken von Boden, Arbeit und Kapital beruhte. ${ }^{308}$

Einen weiteren Angriffspunkt bildete die physiokratische Steuerabwälzungstheorie. Die Kameralisten erkannten, dass die Preisgestaltung von den Konkurrenzbedingungen des Marktes abhing und der Landwirt auch wegen der Verderblichkeit seiner Produkte - anders als der Kaufmann oder Fabrikant - unter Verkaufszwang stand. Dieser fehlende Einfluss auf das Preisgefüge zwang den Landwirt, auferlegte Steuern auch wirtschaftlich zu tragen. ${ }^{309}$ Damit traf die „einzige Steuer“ die Ärmsten der Armen am härtesten. ${ }^{310}$

Die abschließende Idee einer Einheitssteuer im Kameralismus verband die Befürwortung einer allgemeinen Einkommensteuer mit der Erkenntnis, dass es besser wäre, die Gerechtigkeit der Besteuerung durch ein kompliziertes System von

306 Ebd., S. $198 \mathrm{f}$.

307 Dollfus, Roger: Über die Idee der einzigen Steuer, Basel 1897, S. 48-50.

308 Jenetzky, Johannes: System und Entwicklung, S. 135.

309 Justi, Johann Heinrich Gottlob von: Staatswirthschaft oder Systematische Abhandlung aller Oekonomischen und Cameral=Wissenschaften, die zur Regierung eines Landes erfordert werden, Zweyter Theil, Welcher die Lehre von dem vernünftigen Gebrauche des Vermögens des Staats, und mithin die eigentliche Cameral- oder Finanz-Wissenschaft in sich begreift, (1755), 2. Aufl., Leipzig 1758, S. $361 \mathrm{f}$.

310 Eschenmayer, Philipp Christoph Heinrich: Vorschlag zu einem einfachen Steuer-Systeme, Heidelberg 1808, S. 15. 
Normen zu schaffen als die erforderliche Gerechtigkeit durch ein bewusst einfach gestaltetes Normensystem zu verletzen. ${ }^{311}$

\subsubsection{Die Fundamentalprinzipien gerechter Besteuerung}

Nach Ansicht der Kameralisten umfasste die Staatshoheitsgewalt nur diejenigen Berechtigungen, deren Wahrnehmung zum Erreichen des gesellschaftlichen Endzwecks notwendig und dienlich war. ${ }^{312}$ Interpretierte man die Staatsmacht als zweckgerichtet, so galt es nur noch, den Inhalt und die Grenzen der staatlichen Rechte zu bestimmen. Den deutschen Kameralisten kam das Verdienst zu, sich diesbezüglich mit der Aufstellung von Grundsätzen, Prinzipien, Maximen und Postulaten für die Gestaltung der Steuern eingesetzt zu haben. ${ }^{313}$

Die vier bekannten Steuerregeln von Adam Smith (Verhältnismäßigkeit, Bestimmtheit, Bequemlichkeit und Billigkeit der Besteuerung) ${ }^{314}$ waren daher keineswegs etwas Neues. Sie waren lediglich eine Wiederholung dessen, was in der kameralistischen Literatur durchaus bekannt und geläufig war. ${ }^{315}$

Die Entwicklung der Grundsätze der Besteuerung waren eine eigenständige Leistung der kameralistischen Finanzwissenschaft, die nach Originalität und Folgewirkung ohne Vergleich dasteht. ${ }^{316}$ Aufbau, Entwicklung und Zweck entsprachen den rechtsethischen Forderungen der Aufklärungsphilosophen und werden auch in heutiger Zeit noch mit geringen Nuancen als steuerpolitische Ideale mit Verfassungsauftrag anerkannt. ${ }^{317}$

Die Eigenart dieses Evolutionsprozesses ist darin zu sehen, dass die Prinzipien in den Anfängen des Steuerrechts kaum in den vorhandenen Steuersystemen berücksichtigt wurden. Im Verlauf des 19. Jahrhunderts jedoch gelang es dann, kontinuierlich die Steuergesetze inhaltlich den aufgestellten Prinzipien anzu-

311 Kessler, Heinrich: Finanzsystem nach den Verhältnissen Württembergs, Stuttgart 1821, S.33.

312 Justi, Johann Heinrich Gottlob von: Staatswirthschaft oder Systematische Abhandlung aller Oekonomischen und Cameral=Wissenschaften, die zur Regierung eines Landes erfordert werden. Erster Theil, Welcher die Lehre von Erhaltung und Vermehrung des Vermögens des Staats, und mithin die Staatskunst, die Poicey- und Commercien-Wissenschaft nebst der Haushaltungskunst in sich begreift, (1755), 2. Aufl., Leipzig 1758, S. 59 f.; Ders., Staatswirthschaft. Zweyter Theil, S. 3 f.

313 Gerloff, Wilhelm: Steuerwirtschaftslehre, in: Gerloff, Wilhelm/Meisel, Franz (Hrsg.): Handbuch der Finanzwissenschaft, Tübingen 1926, S. 436-489, S. 450.

314 Vgl. hierzu Kapitel 5.4.1.

315 Gerloff, Wilhelm: Steuerwirtschaftslehre, S. 450.

316 Jenetzky, Johannes: System und Entwicklung, S. 99.

317 Ebd., S.99. 
nähern. Heute stimmen diese kameralistischen Besteuerungsgrundsätze mit dem geltenden Steuerrecht überein, soweit es sich nicht um durch die Zeit überholte Grundsätze handelt. ${ }^{318}$

\subsection{Die frühe Kameralepoche (ca. 1650-1730)}

Die frühen Kameralisten gingen dazu über, grundsätzliche Vorstellungen zur abstrakten und generellen Ausgestaltung der Steuern zu erfassen und diese zu einer Art „Allgemeinem Teil“ der Steuerlehre zusammenzustellen. ${ }^{319}$ Diese „Generalregeln“320 waren steuerpolitische Ideale und Maßstäbe, die in den Gesetzen oft genug keine Entsprechung fanden, gleichwohl aber von den Kameralisten mit dem Verlangen nach unbedingter Beachtung und Verwirklichung verfolgt wurden. ${ }^{321}$

Diese Steuerprinzipien waren im Wesentlichen Gerechtigkeitspostulate, stellten aber auch wirtschafts- und finanzpolitische Erwägungen dar. Die Forderung nach Gleichheit der Besteuerung wurde als eine elementare Gerechtigkeitsregel mit unabdingbarem Geltungsanspruch aufgestellt. Die Kameralisten unterschieden im Laufe der Zeit mehrere zu selbstständigen Grundsätzen erhobene Betrachtungsweisen, die den Gehalt des Gleichheitspostulats bestimmten. ${ }^{322}$

Das Allgemeinheitspostulat wurde aus drei verschiedenen Überlegungen heraus begründet: Der individualistische Ansatz basierte erstens auf dem Standpunkt, dass alle Menschen aus der Verwendung der Steuergelder ein gleiches Maß an Schutz genössen und deshalb gehalten sind, in gleicher Weise zu diesen Schutzkosten beizusteuern. Zweitens wies Lith auf die wirtschaftliche Abhängigkeit von individueller und kollektiver Leistungskraft hin und schloss volks- und gesamtwirtschaftliche Überlegungen mit ein. ${ }^{323}$ Er sah die Allgemeinheit der Besteuerung als ein Wesensmerkmal jeder Steuer an und forderte dementsprechend eine Besteuerung ohne

318 Ebd., S.99f.

319 Schulz, Hermann: Das System und die Prinzipien der Einkünfte, S. $114 \mathrm{f}$.

320 Pescherinus, Carl Ferdinand: Politische Gedanken über die bisher zwar ungebräuchlichen aber doch dem Lande und Fürsten höchstersprieslichen Gerneralzehenden. Worinn derselben Vollkommenheit erwiesen, die Beschaffenheit der Anlagen und die Unzulänglichkeit derjenigen, so bisher gebrauchet worden, gezeiget, auch worauf die Glückseligkeit eines Staats beruhe, demonstriret wird. Beigabe zu Schröder, Wilhelm von: Fürstliche Schatz- und Rentkammer, nebst seinem Tractat vom Goldmachen, Königsberg/Leipzig 1752, S. 485-622, S. 518.

321 Schulz, Hermann: Das System und die Prinzipien der Einkünfte, S. 115.

322 Ebd., S. $116 \mathrm{ff}$.

323 Lith, Johann Wilhelm von der: Politische Betrachtungen über die verschiedenen Arten der Steuern, Breslau 1751, S. 8 f. 
Ansehen der Person. ${ }^{324}$ Die dritte Überlegung stammte von den frühen Kameralisten, die in der Akzise bzw. der Praxis der Akzisebesteuerung den Ansatz eines privilegienfreien Kurses gegenüber dem Adel und dem Klerus sahen, der diese Kreise überhaupt in die Steuerpflicht mit einbezog. Die späteren Kameralisten erkannten die Fragwürdigkeit einer solchen einseitigen Verbrauchsbesteuerung und setzten sich daher für die durchgehende, direkte Besteuerung ein. ${ }^{325}$

Wie zum Allgemeinheitspostulat entwickelten die Kameralisten auch zum Prinzip materiell gleicher Belastung aller Steuerpflichtigen die These, dass sich die Beitragslast eines jeden nach dem Maß der Vorteile zu richten hätte, die dieser unter dem Schutz des Staates genösse oder genießen könnte. Endgültige Antworten darauf, ob die Höhe der verursachten Kosten (Kostentheorie) oder der Wert des dem Einzelnen zufließenden Nutzens (Nutzentheorie) zugrunde gelegt werden sollten, wurden jedoch nicht gefunden. ${ }^{326}$

Die Kameralisten bekannten sich zum Grundsatz der „Mäßigkeit“, d. h. zum Verbot übermäßiger Abgaben. Der inhaltlich vage Mäßigkeitsgrundsatz wurde ergänzt durch das konkrete Verbot der Steuerbelastung jenseits der „Einkünfte“. Damit legten die Kameralisten die Basis für eine Besteuerung nach der Leistungsfähigkeit, die die Steuerkraft eines jeden Pflichtigen berücksichtigt, und betonten, dass der erzielte Ertrag bzw. Gewinn als Ausdruck dieser Kraft das richtige Besteuerungsgut wäre. ${ }^{327}$ Ein Bürger, der im Verhältnis zu den Mitbürgern bei gleichen Erträgen mehr oder weniger als sie an Steuer zu entrichten hätte, wäre den Autoren zufolge über das rechte Maß belastet bzw. befreit. Zu den Wegbereitern dieses Prinzips der Besteuerung nach der Leistungsfähigkeit zählten Veit Ludwig von Seckendorff und Johann Daniel Eulner. Seckendorf führte 1720 hierzu wie folgt aus:

So pflegen die christlichen hohen Obrigkeiten, welche dieses rechts fähig sind [...], eine rechtmäßige billige proportion damit zu halten, niemanden deswegen zur ungebühr vor andern zu beschweren, oder zu befreyen, sondern sich damit, als christliche und milde Regenten zu erweisen, welche ihre macht nicht zu unterdrückung, nachtheil

324 Lith, Johann Wilhelm von der: Neue vollständig erwiesene Abhandlung von denen Steuern und deren vortheilhafter Einrichtung in einem Lande nach den Grundsätzen einer wahren die Verbesserung der Macht des Regenten und die Glückseligkeit seiner Unterthanen wirkenden Staatskunst, Ulm 1766, S. 168.

325 Schulz, Hermann: Das System und die Prinzipien der Einkünfte, S. 126.

326 Ebd., S. 127.

327 „Daß derjenige, welcher den genieß eines guts oder anderer einkunfft hat, auch die beschwerungen nach rechter und gleicher proportion, wie andere seine mit=unterthanen nach dem ihrigen tragen mögen." Siehe Seckendorff, Veit Ludwig von: Teutscher Fürsten-Staat samt des Seel. Herrn Autoris Zugabe sonderbarer und wichtiger Materialien, Jena 1720, S. 496. 
und quaal, sondern zu schutz, rettung und erquickung der armen bedrängten unterthanen zu gebrauchen haben. ${ }^{328}$

Deutlicher forderte Eulner 1741: „Steuren und Tributen nur von der Nutzbarkeit und Einkommen eines jeden steuerbaren Stücks“ zu nehmen, d.h. „von allen [...] Renthen und Einkommen“, sodass „keiner vor dem andern beschweret werde, sondern ein jeder nach seinem Vermögen neben dem andern gleiche Anlage und Steuer gebe“. 329

Als eigenständiges Prinzip wurde die Besteuerung nach der individuellen Leistungsfähigkeit zur Mitte des 18. Jahrhunderts ausformuliert. Johann Heinrich Gottlob Justi (1717-1771) begründete dies wie folgt:

\begin{abstract}
Der erste und hauptsächlichste Grundsatz, den man bey denen Steuern und Abgaben unaufhörlich vor Augen haben muß ist, daß sich die Unterthanen im Stande befinden müssen, solche leisten zu können. Sie befinden sich aber alsdenn nur im Stande, solches zu thun, wenn sie die Abgaben tragen können, ohne Abbruch ihrer Nothdurft, und ohne den Hauptstamm ihres Vermögens anzugreifen. Abgaben, die sich nicht in diese Gränzen einschließen, verdienen diesen Nahmen nicht; sie sind tyrannische Erpressungen und ein gewaltsamer Raub des Vermögens der Unterthanen. ${ }^{330}$
\end{abstract}

Die historisch gewachsenen Steuerfreiheiten lehnten die Kameralisten - nach dem Verfassungsverständnis der Aufklärungszeit - nahezu einhellig ab. Josef von Sonnenfels bezeichnete 1771 die vorhandenen Steuerfreiheiten als „Diebstahl am gemeinen Wesen, oder an den Mitbürgern“. ${ }^{331}$ Auch Seckendorff forderte für die historisch begründeten Steuerbefreiten die Steuerpflicht. ${ }^{332}$ Den Adel traf die Verpflichtung, seine nicht mehr ausgeübten Kriegsdienste durch Steuerzahlungen auszugleichen,

328 Ebd., S. 507.

329 Eulner, Johann Daniel: Practische Vorschläge, welcher gestalt Steuer und Contribution zum Nutzen eines Landesherrn und ohne Nachtheil der Unterthanen nach Anleitung der Reichsabschiede einzurichten seyn, zweyte mit Anmerckungen und einem Appendice vermehrte Auflage: wie Contribution und Accise gegen einander balanciren / und wie weit solche neben der Contribution ohne Nachtheil der Unterthanen bestehen könne. Durch einen neu ausgefundenen Modum Accisandi mit nöthiger Anweisung versehen und practisch ausgeführet, Marburg 1741, S.91 f.

330 Justi, Johann Heinrich Gottlob von: Ausführliche Abhandlung, S. 23, § 10.

331 Sonnenfels, Josef von: Grundsätze der Polizey, Handlung und Finanz. Zu dem Leitfaden des politischen Studiums (1771), 5. Aufl., Wien 1787, S. 165.

332 Seckendorff, Veit Ludwig von: Teutscher Fürsten-Staat, S. 500. 
da das neuzeitliche Kriegswesen aus dem Steueraufkommen bestritten wurde. ${ }^{333}$ Der Geistlichkeit wurde jede Berechtigung auf Steuerfreiheit abgesprochen. ${ }^{334}$

Der entscheidende Durchbruch im Denken der kameralistischen Schriftsteller lag im Wechsel des Verständnisses steuerlicher Rechtsbeziehungen vom Privatrecht zum öffentlichen Recht. Justi, der auch eine Schlüsselstellung für die im Kameralismus entwickelten Besteuerungsprinzipien einnahm, leitete als Erster aus den allgemeinen staatswirtschaftlichen Grundsätzen das Recht des Regenten ab, alle historisch gewachsenen Steuerfreiheiten aufzuheben: „denn die Glückseligkeit des Staats ist dessen höchstes Gesetz. “335

Die Aufhebung der standesbedingten Steuerfreiheiten wurde von allen Kameralisten gewünscht; Mitte des 19. Jahrhunderts wurden diese Forderungen durch die Gesetzgebung umgesetzt. Inwieweit der Kameralismus an diesen Gesetzgebungsverfahren mitgewirkt hat, ist schwer nachzuvollziehen; der aufklärerische Zeitgeist wurde wahrscheinlich von den Vertretern der Lehre und Wissenschaft mit beeinflusst. ${ }^{336}$

Unabhängig von der Kritik an den historisch begründeten Steuerfreiheiten entwickelten die kameralistischen Autoren spezielle Steuerfreiheiten, die aus Gerechtigkeitsgründen oder wegen der wirtschaftlichen Notwendigkeit als zweckmäßig gefordert wurden.$^{377}$ Es erforderte z. B. keiner besonderen Begründung, Hospitäler, Armenhäuser und Glaubensstiftungen von allen Steuern zu befreien. ${ }^{338}$ Auch für diejenigen, die keinen Gewinn erwirtschafteten, entstand von vornherein keine Steuerpflicht. Bedürftige, Vermögenslose und arme Leute galten als nicht belastbar und waren daher von der Entrichtung der Abgaben ganz freizustellen. ${ }^{339}$ „Erhalt des Notbedarfs“, „Schonung der Armen“ und Besteuerung erst ab „Überschreiten des Existenzminimums“ waren die Stichworte, die die unterschiedlichen Begrün-

333 Justi, Johann Heinrich Gottlob von: Staatswirthschaft, Erster Theil, S. 428.

334 Ebd., S. $426 \mathrm{f}$.

335 Ebd., S. 45.

336 Sensburg, Ernst Philipp Freiherr von: Pragmatische Untersuchung des Ursprunges und der Ausbildung alter Abgaben und Steuern zur Vorbereitung eines gleichheitlichern, und repräsentativen Verfassungen angemessenern Abgaben-Systems, Erlangen 1823, S. 137.

337 Jakob, Ludwig Heinrich von: Die Staatsfinanzwissenschaft. Theoretisch und praktisch dargestellt und erläutert durch Beyspiele aus der neuern Finanzgeschichte europäischer Staaten, Bd. 2, Halle 1821, $\$ \$ 1151-1174$.

338 Kröncke, Claus: Über die Grundsätze einer gerechten Besteuerung aus: Abhandlungen über staatswirthschaftliche Gegenstände, Vierter Theil, Heidelberg 1819, S. 30.

339 Jakob, Ludwig Heinrich von: Die Staatsfinanzwissenschaft, Bd. 2, § 1161. 
dungsansätze zur Festlegung der absoluten Steuerbelastungsgrenze und damit die Untergrenze der Besteuerung markierten. ${ }^{340}$

Neben den Gerechtigkeitsmaximen entwickelten die Kameralisten Grundsätze des Steuerrechts aus wirtschafts- und finanzpolitischen Erwägungen, die zum Teil um die Mitte des 19. Jahrhunderts von John Stuart Mill den Namen der „klassischen Maximen" erhielten. Als eigenständige Maximen wurden neben dem Verbot der Doppelbesteuerung, dem Grundsatz der Ertrags- bzw. Gewinnbesteuerung, dem Grundsatz der Wirtschaftsfreundlichkeit und Wirtschaftsförderlichkeit der Steuern, sowie dem Grundsatz der Vermögensgeheimhaltung, die folgenden Grundsätze formuliert: ${ }^{341}$

Präzise Steuerbestimmung sollten für die Untertanen Klarheit schaffen: „Jedermann muß so wohl den Grund der Abgaben wissen, als die Größe und Quantität derselben, damit er nicht der Willkühr und denen Bedrückungen der Einnahme bedienten, ausgesetzet ist". ${ }^{342}$

Außerdem müsse man den Abgaben „eine solche Einrichtung geben, daß sie auf die bequemste und leichteste Art und mit so wenigen Kosten als es immer möglich ist, so wohl auf Seiten des Staats als der Unterthanen gehoben werden können. “"343 Damit wurde 1762 das klassische Bequemlichkeitspostulat vorweggenommen, das in der nachkameralen Zeit als geistige Errungenschaft Adam Smiths 1776 angesehen wurde. ${ }^{344}$

Schon vor Adam Smith hatten Justi, Bergius und Sonnenfels in Deutschland den Grundsatz der Wohlfeilheit der Besteuerung zu einem weiteren selbstständigen Besteuerungsprinzip erhoben. ${ }^{345}$

Aus diesem Postulat leiteten die Kameralisten den Grundsatz der Besteuerung nach dem Zustand des Landes und der Steuerpflichtigen ab. Steuern sollten ohne Schaden für die Untertanen erbracht und auch ohne Nachteil für die Wirtschaft des Landes erhoben werden können. Aber nicht nur die Höhe der gesamten Steu-

340 Schulz, Hermann: Das System und die Prinzipien der Einkünfte, S. 134.

341 Ebd., S. $134 \mathrm{ff}$.

342 Justi, Johann Heinrich Gottlob von: Ausführliche Abhandlung, S. 42.

343 Ebd., S. 42.

344 Smith, Adam: Der Wohlstand der Nationen, S. 704.

345 Justi, Johann Heinrich Gottlob von: Staatswirthschaft, Zweyter Theil, S. $306 \mathrm{ff}$.; Bergius, Johann Heinrich Ludwig: Policey- und Cameral-Magazin in welchem nach alphabetischer Ordnung die vornehmsten und wichtigsten bey dem Policey- und Cameralwesen vorkommende Materien nach richtigen und vernünftigen Grundsätzen practisch abgehandelt und durch landesherrliche Gesetze und hin und wieder wirklich gemachte Einrichtungen erläutert werden, Bd. 1, Frankfurt am Main 1767, S. 3 ff. 
ern, sondern auch ihre Erhebung und Verwendung müssten dabei stets mit dem Herrschaftszweck in Übereinstimmung stehen. ${ }^{346}$

Eine umfassende „gerechte" und „kluge“ Steuerordnung erforderte die Einheit und Widerspruchsfreiheit einer Mehrheit von Steuern. Justi sah in der Vielzahl von Steuern etwas Unvollkommenes, doch waren die Kameralisten Realisten genug, diese Erkenntnis zu nutzen, um möglichst weitgehend die aufgestellten Besteuerungsgrundsätze in einer Steuerordnung zu berücksichtigen. ${ }^{347}$

\subsection{Die spätere Kameralepoche (ca. 1730-1835)}

Mit den späteren Kameralisten erreichten die Besteuerungsgrundsätze den hohen Grad steuerlicher Ideale, die der Konkretisierung bedurften. ${ }^{348}$ Viele früher strittige konkrete Einzelfragen hatten sich historisch überholt oder konnten gelöst werden. In der erreichten Form konnten diese abstrahierenden Maximen als unbestrittene Erkenntnisse Eingang in die Finanzwissenschaft finden. Hierin lag die bleibende Leistung der kameralistischen Steuerliteratur. ${ }^{349}$ Der Historiker Friedrich Meinecke hat es einmal so ausgedrückt:

Mochte auch den Staatsmännern selber nichts Neues damit gelehrt werden, so war doch neu, daß es gelehrt wurde. Denn erst durch die Erfassung als Prinzip erhalten die historischen Tendenzen ihre volle Stoßkraft und steigern sich zu dem, was man Idee nennen kann. ${ }^{350}$

Während die früheren Kameralisten die Besteuerungsgrundsätze mehr willkürlich als planvoll aneinandergereiht hatten, gingen die späten dazu über, die „obersten Prinzipien der positiven Gesetzgebung" in solche der Justizgesetze, der Polizeygesetze sowie der Finanzgesetze zu gliedern. ${ }^{351}$

Gegen Ende der Kameralzeit begegnen uns die Besteuerungsgrundsätze als steuerpolitische Ideale mit dem Anspruch auf vollständige Verwirklichung in der Steuergesetzgebung und Steuerverwaltung. Gleichzeitig relativierten die Autoren jedoch den unmittelbaren Nutzwert der Maximen, da bei der Durchführung die-

346 Justi, Johann Heinrich Gottlob von: Staatswirthschaft, Zweyter Theil, S. $311 \mathrm{ff}$.

347 Schulz, Hermann: Das System und die Prinzipien der Einkünfte, S. $151 \mathrm{f}$.

348 Jenetzky, Johannes: System und Entwicklung, S. $121 \mathrm{f}$.

349 Ebd., S. 122.

350 Meinecke, Friedrich: Die Idee der Staatsräson in der neueren Geschichte, München/ Berlin 1924, S. 49.

351 Jakob, Ludwig Heinrich von: Einleitung in das Studium der Staatswissenschaften als Leitfaden für seine Vorlesungen ausgearbeitet, Bd. 1, Halle 1819, S. $254 \mathrm{ff}$. und S. $264 \mathrm{f}$. 
ser Ziele viel Unkenntnis und Uneinigkeit bestünde. ${ }^{352}$ Nahezu geschlossen und nachhaltig jedoch äußerten sich die Kameralisten zur Reform der Steuern unter den Gesichtspunkten der Begrenzung und gerechten Verteilung der Steuerlast, die oft an erster Stelle des Kataloges der Steuerprinzipien standen: ${ }^{353}$

Das Gebot der Beschränkung der Steuern auf das staatsnotwendige Maß - auf die unvermeidlichen Ausgaben des Staates - galt als Maxime der „Gerechtigkeit“. Denn „mehr als die Noth soll nicht gesteuert werden. [...] Wo daher Steuern als Ergänzung der übrigen Staatseinkünfte zur Befriedigung der Staatsbedürfnisse notwendig sind, [...] da darf man sie auch als gerechtfertiget ansehen." ${ }^{354}$ Die späten Kameralisten reduzierten das Gebot der absoluten Beschränkung auf das Notwendigste, auf ein Minimum, da auf die Leistungskraft des Volkes Rücksicht zu nehmen wäre. ${ }^{355}$

Das Mäßigkeitstheorem - die Untertanen sollten nur mit mäßigen Steuern belegt werden dürfen, „denn schon aus den Grundsätzen eines natürlichen Staatsrechts folgt ein gewisses Maas der Steuern“356 - war zu unbestimmt, weshalb es durch das Gebot des Substanzschutzes und das Prinzip der Erhaltung der steuerlichen Leistungsfähigkeit abgelöst wurde. Das Verbot „drückender Auflagen“ war in den Augen der Kameralisten nun zu wenig aussagekräftig und fand wie das Prinzip der Mäßigkeit der Steuern bald keine Aufnahme mehr in den Katalog der Steuerprinzipien.

Das Gebot des Substanzschutzes hingegen wurde auf mehrfache Weise begründet. Insbesondere sollte die Beitragsfähigkeit der Bürger erhalten bleiben ${ }^{357}$ und „nie das steuerbare Kapital selbst" angegriffen werden dürfen. ${ }^{358}$ Auch den Grundsatz der nur teilweisen Gewinnbesteuerung wussten die Autoren des Kameralismus mehrfach zu begründen. Beraubte man die Bürger der Früchte ihres Fleißes, so entfiele bei ihnen jeder „Ansporn“, und die Neigung, das Land zu verlassen, machte sich breit.

352 Schulz, Hermann: Das System und die Prinzipien der Einkünfte, S. 356.

353 Ebd., $356 \mathrm{ff}$.

354 Rau, Karl Heinrich: Lehrbuch der politischen Oekonomie, Bd. 3: Grundsätze der Finanzwissenschaft, Erste Abtheilung, 3. Aufl., Leipzig/Heidelberg 1855, S. 343 f.

355 Jung (genannt Stilling), Johann Heinrich: Lehrbuch der Finanz-Wissenschaft, Leipzig 1789, ND Wiesbaden 1978, S. 3 f., $\$ \$ 7$ und 8.

356 Lang, Karl Heinrich von: Historische Entwicklung, S. 206.

357 Sonnenfels, Joseph von: Grundsätze, S. 199-202.

358 Sturm, Karl Christian Gottlob: Grundlinien einer Encyklopaedie der Kameralwissenschaften, Jena 1807, S. 334 . 
Dem Rückgang des Erwerbs würden der Verfall des Vermögens und damit auch der Niedergang des Staates folgen. ${ }^{359}$

Die späten Kameralisten verstanden unter zureichenden Gesamtsteuereinkünften,

daß diejenigen, welche im Staate leben, die Segnungen der Staatsverbindung empfinden und die Vortheile mancher einzelnen Anstalten genießen, dafür auch an den Beschwerden und Aufopferungen Theil nehmen müssen, ohne welche der Staat seine volle Wirksamkeit nicht äußern könnte. ${ }^{360}$

Aus der Erkenntnis, dass eine stetig zunehmende Staatstätigkeit auch steigende Abgaben verursacht, wurde die Pflicht sowohl für die Herrschenden als auch für die Untertanen abgeleitet, stetig steigende Abgaben zu fordern und entsprechend auch zu entrichten. ${ }^{361}$

Dem Prinzip der Steuergleichheit maßen sowohl die frühen als auch die späten Kameralisten den höchsten Stellenwert zu. Dabei erkannten sie jedoch, dass es äußerst schwierig war, diese Forderung nach Gerechtigkeit in die Praxis umzusetzen. ${ }^{362}$ Die Autoren führten ungerechtfertigte Steuerbefreiungen und Unzulänglichkeiten der verschiedenen Steuerarten als Ursachen für die tatsächlichen Steuerungleichheiten an. ${ }^{363}$

Ohne Ausnahme verstanden die Kameralisten unter dem bis heute gültigen Prinzip der Allgemeinheit der Steuern die unverrückbare Maßgabe, dass alle Staatsbürger steuerpflichtig seien. Das Gebot der „Gleichmäßigkeit“ oder Gleichförmigkeit der Steuern stellte sich für die Autoren wie folgt dar: „alle Bürger sollen

359 Lith, Johann Wilhelm von der: Neue vollständige erwiesene Abhandlung, S. 13 f.

360 Rau, Karl Heinrich: Lehrbuch der politischen Ökonomie, Erste Abtheilung, S. 343.

361 Lueder, August Ferdinand: Über Nationalindustrie und Staatswirthschaft, nach Adam Smith bearbeitet, Bd. 1-3, Berlin 1800-1804, S. 506.

362 Justi, Johann Heinrich Gottlob von: System des Finanzwesens. Nach vernünftigen aus dem Endzweck der bürgerlichen Gesellschaften und aus der Natur aller Quellen der Einkünfte des Staats hergeleiteten Grundsätzen und Regeln, Halle an der Saale 1766, ND Aalen 1969, S.364; Jung (genannt Stilling), Johann Heinrich: Lehrbuch der Finanz-Wissenschaft, S. 215.

363 Lips, Alexander: Ueber die allein wahre und einzige Steuer die Einkommen-Taxe und ihre Ausführbarkeit. Ein Versuch die Kosten der General-Landes-Vermessung zum Behuf der General-Steuer-Rektifikazion, das Steuerprovisorium und die Mehrheit der Steuern überhaupt zu umgehen, und an deren Stelle eine allgemeine, einzige, gleiche, gerechte, alle treffende, keinen drückende, kraftvolle, und in ihrer Erhebung höchst einfache Nazionalauflage zu setzen, Erlangen 1812, S. 5. 
nach gleichen Regeln und nach einem gleichen in ihrem Verhältniß zum Staate begründeten Maaßstabe beigezogen werden. “" ${ }^{364}$

Die stets gleichbleibende Begründung für die Steuergleichheit war die Garantie der bürgerlichen Freiheit und des Schutzes vor Angriffen von innen und von außen. Justi führte dieses Staatsziel wie folgt aus:

da sie alle gleichen Schutz genießen; so müssen sie auch zu dem großen Aufwand des Staats gleichmäßig Beytrag thun [...] und da derjenige, welcher ein größeres Vermögen besitzet, auch einen größern Schutz genießet: so kommt eben diese gerechte Gleichheit darauf an, daß ein jeder Unterthan nach Proportion seines Vermögens seinen Beytrag zu dem Aufwand des Staats leistet. ${ }^{365}$

Für die Kameralisten stand fest, dass die Steuern in der Staatlichkeit der Gesellschaft begründet waren und daher jeder Staatsbürger für die Vorteile, die ihm aus der Staatsbürgerschaft erwuchsen, Steuern zu entrichten hatte. ${ }^{366}$ Einig waren sich die Kameralisten auch darin, dass Steuerbefreiungen als ungerechtfertigt gegenüber anderen Bürgern waren, mag es sich dabei um eine generelle Steuerbefreiung oder um Steuerfreiheiten einzelner Personen aus alten Verträgen oder Privilegien handeln. ${ }^{367}$ Keine Einigkeit bestand jedoch darin, ob die Steuertarife proportional oder progressiv zu gestalten waren. Sprachen sich die Autoren des Frühkameralismus noch eindeutig für eine „rechtmäßige und billige Proportionalität“ bei der Besteuerung aus, ${ }^{368}$ so zog die Steuerpraxis der wirtschaftlich fortgeschrittenen

364 Rau, Karl Heinrich: Lehrbuch der politischen Ökonomie, Erste Abtheilung, S. 344. Nach Justi sollten die „Abgaben mit einer vollkommenen Gleichheit und gerechten Verhältniß den Unterthanen aufgeleget werden“; Justi, Johann Heinrich Gottlob von: Ausführliche Abhandlung, S.26f.

365 Ebd., S. 27.

366 Ebd., S. 27; Jung (genannt Stilling) Johann Heinrich: Lehrbuch der Finanz-Wissenschaft, $\$ \$ 5,6,7$ und 8; Wilke stellt heraus, dass Sonnenfels die Tendenz der tatsächlichen Entwicklung des Finanzwesens der deutschen Staaten richtig erfasst habe und daher die Steuern als regelmäßige staatliche Einnahmequelle behandelte. Damit beginne sich die wissenschaftliche Behandlung des Finanzwesens von der mehr privatwirtschaftlichen Betrachtungsweise über die Domänen- und Regalienwirtschaft zu lösen und zu einer staatswirtschaftlichen Erörterung finanzieller Probleme vorzudringen; Wilke, Gustav: Die Entwicklung der Theorie, S. 11.

367 Rüdiger, Johann Christian Christoph: Anfangsgründe der allgemeinen Staatslehre mit einem kurzen Lehrbegriff der ökonomischen Policey, Halle 1795, S. 130.

368 Eulner, Johann Daniel: Practische Vorschläge, S. 792. 
westeuropäischen Staaten, insbesondere Englands, die Idee der Steuerprogression nach sich. ${ }^{369}$

Justi entwickelte als Erster das folgende Beispiel einer gemischten Vermögensund Einkommensbesteuerung zweier unterschiedlich begüterter Kaufleute: ${ }^{370}$

\begin{tabular}{|c|c|c|c|}
\hline & Kfm. Martin & Kfm. Christoph & prop.Verhältnis \\
\hline Vermögen & 20.000 Thaler & 60.000 Thaler & $1: 3$ \\
\hline Gewinn (pro Jahr) & 3.000 Thaler & 9. 000 Thaler & \\
\hline Haushaltskosten ${ }^{\star}$ & 2.000 Thaler & 2.000 Thaler & \\
\hline Verbleibender Rest & 1.000 Thaler & 7.000 Thaler & \\
\hline $\begin{array}{l}1 \% \text { Steuer auf das Vermögen } \\
\text { (proportional) }\end{array}$ & 200 Thaler & 600 Thaler & $1: 3$ \\
\hline Verbleibender Rest & 800 Thaler & 6.400 Thaler & $1: 8$ \\
\hline \multicolumn{4}{|c|}{${ }^{\star}$ Kosten der Haushaltung, Familie und Bedienung } \\
\hline
\end{tabular}

Kaufmann Martin behielt nur 800 Thaler übrig, Kaufmann Christoph hingegen hätte 6.400 Thaler zur Vergrößerung seines Vermögens übrig, das wäre achtmal mehr, obwohl er nur das Dreifache an Steuern entrichtet.

Dieses einfache Beispiel zeigt hervorragend, wie unbefriedigend die proportionale Besteuerung ist, was Justi veranlasste, auf die ungerechte Proportionalität bei der Steuerbelastung hinzuweisen, bei der die Reichen nicht entsprechend erfasst würden und die ganze Last auf den Schultern des Mittelstandes ruhe. ${ }^{371}$

Von Justi im Jahr 1762 ausgehend wurde die progressive Steueridee neben der proportionalen Besteuerung diskutiert. Am Ende der Kameralzeit war der Gedanke der progressiven Besteuerung vorherrschend; der Progressionsmaßstab blieb jedoch eine Frage der Finanzpolitik. ${ }^{372}$

Der Grundsatz der Sicherheit der Einnahmen beruhte auf dem Grundanliegen, dass in der Staatsmacht das Recht und die Pflicht begründet war, sich die Mittel zu verschaffen, welche zur Realisierung der Staatszwecke „gewiss und sowohl an Quantität und Qualität so bestimmt sind, dass keine Zweydeutigkeit dabey Statt

369 Raumer, Friedrich von: Das Brittische Besteuerungs-System, insbesondere die Einkommensteuer, dargestellt, mit Hinsicht auf die in der preußischen Monarchie zu treffenden Einrichtungen, Berlin 1810, S. 237.

370 Justi, Johann Heinrich Gottlob von: Ausführliche Abhandlung, S. 27 f.

371 Ebd., S.28.

372 Jenetzky, Johannes: System und Entwicklung, S. 142-144. 
findet. ${ }^{\left({ }^{373}\right.}$ Im Grundsatz der Bestimmtheit der Steuern und der Transparenz der staatlichen Einkünfte findet sich eine allgemein anerkannte Forderung der Kameralisten wieder, die lautete, dass jeder Steuerpflichtige die Steuerlast nach Art und Weise der Zeit sowie der Höhe nach im Voraus genau bestimmen können müsse. ${ }^{374}$ Um die Besteuerung unmerklich erscheinen zu lassen, musste „die Erhebung der Abgaben [...] bequem und leicht eingerichtet seyn ", ${ }^{375}$ und um die Steuererhebungskosten so gering wie möglich („wohlfeil“) zu halten, musste die Steuer „leicht und kostenlos ausgemittelt, erhoben und verrechnet werden können “. 376

Die späten Kameralisten betrachteten die Steuergestaltung als vorzügliches Mittel, Staat, Wirtschaft und Gesellschaft nach den wohlfahrtspolizeilichen Vorstellungen zu lenken, zu leiten und zu formen. ${ }^{377}$ Gestützt auf die Überzeugung, dass der Staat in der Regel kein Unrecht tun kann, schlugen die Kameralisten ab der Periode des Hochkameralismus umwälzende Eingriffe in die private Vermögenssphäre durch steuerliche Maßnahmen vor. Die Steuer sollte Luxus und Verschwendung unterdrücken, und Handel, Erzeugung und Gewerbe sollten durch steuerliche Eingriffe je nach Bedarf eingeschränkt oder gefördert werden. ${ }^{378}$

Ein nicht sehr häufig anzutreffender Steuergrundsatz besagte, dass die Verteilung der Steuerlast der Struktur der Staatsverfassung und der Wirtschaft anzupassen wäre. ${ }^{379}$ Ebenfalls selten traf man den Gedanken an, dass „die Unterthanen die Abgaben gleichsam aus eigener Bewegung und mit willigem und freudigem Herzen entrichten". ${ }^{380}$

Aus Gründen der Gerechtigkeit sollten die Steuern so gestaltet sein, dass sie dem Vermögen des Steuerpflichtigen und der Wirtschaft keinen höheren Schaden

373 Jakob, Ludwig Heinrich von: Die Staatsfinanzwissenschaft. Theoretisch und praktisch dargestellt und erläutert durch Beyspiele aus der neuern Finanzgeschichte europäischer Staaten, Bd. 1, Halle 1821, $\$ 463$.

374 Justi, Johann Heinrich Gottlob von: Ausführliche Abhandlung, S. 41; Smith, Adam: Der Wohlstand der Nationen, S. 704.

375 Justi, Johann Heinrich Gottlob von: Ausführliche Abhandlung, S. 42.

376 Lips, Alexander: Deutschlands National-Oekonomie. Ein Versuch zur endlichen Lösung der Frage: wie kann Deutschland zu lohnendem Ackerbau, zu blühender Industrie und wirksamem Handel gelangen?, Gießen 1830, S. 210.

377 Jenetzky, Johannes: System und Entwicklung, S. $84 \mathrm{ff}$.

378 Bensen, D. Heinrich: Materialien zur Polizei-, Kameral- und Finanzpraxis, Bd. 2, Erlangen 1802, S. 428.

379 Justi, Johann Heinrich Gottlob von: Ausführliche Abhandlung, S. 39 f.

380 Bergius, Johann Heinrich Ludwig: Policey- und Cameral-Magazin, S. 4. 
zufügten, als sie dem Staat Gewinn brächten. ${ }^{381}$ Die Maxime, dass das erlangte Wissen der Finanzbediensteten vor den anderen Bürgern geheim zu halten ist, formulierten die späteren Kameralisten, was seine Gültigkeit in Form des Steuergeheimnisses bis heute erhalten hat. ${ }^{382}$

Überwiegend vertraten die Kameralisten den Leitgedanken der harmonisch abgestimmten Steuervielfalt. Die Autoren betonten wiederholt, dass die Einheitssteuer undurchführbar und ökonomisch ungeeignet sei und wegen der verschiedenen persönlichen Verhältnisse zu einer ungleichen Steuerbelastung führe. ${ }^{383}$ Aus der Erkenntnis, dass es viele Quellen des allgemeinen Wohlstandes gibt und direkte und indirekte Steuern Vor- und Nachteile gegenüber der jeweils anderen Besteuerungsform aufweisen, folgerten sie, dass nur ein Mischsystem von verschiedenen Steuern zu einem staatswissenschaftlich gerechten Ergebnis führen könne. ${ }^{384}$

Nach einhelliger Meinung der Kameralisten war die Besteuerung des „Ertrages“ bzw. "Gewinnstes“ ein anzustrebendes Ideal. Über den Begriff des „Gewinnstes“ oder „Ertrages“ bestand sachlich Übereinstimmung, ${ }^{385}$ auch wenn der Begriff mit unterschiedlichen Worten erläutert wurde. Justi definierte diesen wie folgt: „Gewinnst ist der Vortheil, den wir nach Abrechnung unserer angewendeten Kosten und Bemühungen aus einer Sache ziehen. ${ }^{\text {"386 }}$

Die Autoren des Kameralismus erkannten schon früh, dass die Vermögensteuer konsequenterweise auch die Besteuerung ertragsloser Gegenstände erfassen würde und dass letztlich jede Steuer aus dem Einkommen erfolgen müsste. ${ }^{387}$ Gegen die echten Vermögensteuern sprach auch, dass diese die Quelle erschöpfen würden, aus der sie fließen. ${ }^{388}$ Justi betonte besonders die Notwendigkeit, dass die Vermögenssubstanz geschont werden müsse, und befürwortete die Besteuerung des

381 Jakob, Ludwig Heinrich von: Einleitung in das Studium der Staatswissenschaften, S. 370 .

382 Jenetzky, Johannes: System und Entwicklung, S. 205-208.

383 Spaeth, Philipp Jacob: Gedanken über das Steuerwesen im Allgemeinen und über Steuer-Rektifikationen insbesondere, Stuttgart 1806, S. $30 \mathrm{ff}$.

384 Jakob, Ludwig Heinrich von: Die Staatsfinanzwissenschaft, Bd. 1, §686, §\$ 693-705.

385 Jenetzky, Johannes: System und Entwicklung, S. 194.

386 Justi, Johann Heinrich Gottlob von: Staatswirthschaft, Erster Theil, S. 444.

387 „Schonung des Capitales eines Volkes ist also eine nothwendige Anforderung an jedes Steuersystem.“ „Demnach beruht die Steuerfähigkeit der Bürger vorzüglich auf ihrem Einkommen [...], so müssen die Steuern soviel als möglich nach dem Verhältnis des Einkommens vertheilt werden.“ „Das reine Einkommen eines Volkes ist die Quelle, aus der sämmtliche Steuern geschöpft werden müssen.“; Rau, Karl Heinrich: Lehrbuch der politischen Oekonomie, Erste Abtheilung, S. 349 f., S. 352 und S. 355.

388 Lips, Alexander: Ueber die allein wahre und einzige Steuer, S. 14. 
Gewinns, was von den kameralistischen Autoren konsequent befürwortet wurde. Carl Gotthelff Prätorius hatte es 1802 auf den Punkt gebracht:

Abgaben müssen ihrer Natur nach nur vom reinen Ertrage genommen und nie so hoch angelegt werden, dass sie das Kapital des Staatsbürgers weder mittelbar noch unmittelbar angreifen. Dies ist unstreitig der erste und wichtigste Grundsatz. ${ }^{389}$

Auch Philipp Christoph Heinrich Eschenmayer betonte 1813:

Die Besteuerung des Ertrages, des Einkommens der National-Glieder hingegen ist die eigentliche und wahre Basis, die weder das Vermögen, die Capitale, angreifet, noch den Genuss verkümmert, noch die National-Production lähmt. ${ }^{390}$

In seiner „Abhandlung von Domainen, Contributionen, Steuern, Schazungen und Abgaben“ aus dem Jahr 1775 führt Jacob Friedrich Döhler gleichermaßen aus:

Zweytens, müssen die Abgaben und Steuern der Freyheit, dem Credit und den Gewerben der Unterthanen nicht nachtheilig, sondern solche müssen lediglich auf den Gewinnst der Unterthanen gegründet seyn, nachdem es die Natur derer Gegenstände verträget, und diese Abgaben müssen auch einen gerechten Theil des Gewinnsts ausmachen: Der gerechte Theil des Gewinnsts aber muß so proporioniret seyn, das die Unterthanen von dem Üeberrest auch nothdürftig und bequem leben können. ${ }^{391}$

Aus dieser Erkenntnis heraus entwickelte sich ein eindeutiger Trend von der Vermögensteuer als Substanz- zur Einkommensteuer als Ertragsbesteuerung. ${ }^{392}$ Diese schrittweise Evolution begann mit Justi und Sonnenfels. Justi hatte den Ertrag als alleinigen Gegenstand der Besteuerung festgestellt und fühlte sich als Erfinder der eigentlichen Gewerbesteuern, obwohl Eulner sie wissenschaftlich entworfen hatte und sie als direkte Ertragsteuer in der Praxis üblich waren. ${ }^{393}$ Sonnenfels entdeckte die persönliche Arbeitsleistung als eine Einkommen produzierende Kraft ohne Einsatz von sachlichen Vermögenswerten. Dabei betonte er, dass der notwendige Unterhalt des „Wirthschafters“ abzuziehen sei und nur der Rest Maßstab für die Steuerbemessung sein dürfe. Den Anspruch auf den eigenen Unterhalt und auf den

389 Prätorius, Carl Gotthelff: Versuch über das Besteurungswesen, Königsberg 1802, S. 64.

390 Eschenmayer, Philipp Christoph Heinrich: Über die Consumtions-Steuer: eine staatswirthschaftliche Abhandlung, Heidelberg 1813, S. 10.

391 Döhler, Jacob Friedrich: Abhandlung von Domainen, Contributionen, Steuern, Schazungen und Abgaben, Nürnberg 1775, S. 88, \$ 68.

392 Jenetzky, Johannes: System und Entwicklung, S. 192.

393 Eulner, Johann Daniel: Practische Vorschläge, S. 66-70. 
Unterhalt der Familie leitete er von der Pflicht der Selbsterhaltung des Hausvaters ab. ${ }^{394}$

Eines der größten Probleme der Kameralisten bestand darin, den „Gewinnst“ zu ermitteln. Den Autoren war die Problematik einer schematischen Gewinnermittlung aufgrund der steuerlich mangelnden Buchführungsgrundlagen bekannt. ${ }^{395}$ Innerhalb des Systems der Ertragsbesteuerung durchlief der Besteuerungsmodus alle Stadien der Entwicklung von der rohen Kopfsteuer bis zur Versteuerung des bilanziell erzielten Gewinns. Mit dem Ausklang des Kameralismus war jedoch nur ein Teil der Entwicklung durchschritten. ${ }^{396}$

Für die wissenschaftsgeschichtliche Entwicklung kann man drei Epochen unterscheiden, die begrifflich klar zu differenzieren sind, sich jedoch zeitlich überlappen. ${ }^{397}$

Zunächst empfahl man die rein hypothetische schematische Besteuerung des Ertrags - ohne Berücksichtigung der real erzielten Erträge - in Anlehnung an äußerliche Tatbestandsmerkmale, die erfahrungsgemäß Gewinne versprachen. Der Staat hatte zwar kein Recht, von dem Bürger eine Steuererklärung über sein Einkommen zu verlangen, aber er hatte das Recht, die Merkmale des Messens und Bewertens zu bestimmen. Die Behörde konnte nur anhand von äußerlich erkennbaren objektiven Merkmalen den Ertrag des Bürgers schätzen.

Es folgte eine Periode, in der die typisierte Besteuerung diskutiert wurde. Besteuert wurden die üblicherweise erzielten mittleren Durchschnittsgewinne der aus dem 18. Jahrhundert stammenden Ertragsteuern aus den drei Quellen Grund und Boden, Gebäude und Gewerbebetriebe, die den Anforderungen des 19. Jahrhunderts gut zu entsprechen schienen. ${ }^{398}$ Die äußerlichen Merkmale der Besteuerung des durchschnittlich erzielten möglichen Ertrags waren sehr verschieden, weshalb den unterschiedlichen Merkmalskombinationen mithilfe von Einschätzungstafeln Ertragswerte zugeordnet wurden. Diese galten als objektiv ermittelter Maßstab für die gesuchte Leistungsfähigkeit des entsprechenden Objekts. ${ }^{399}$

In der Spätphase der Kameralepoche waren die Kameralisten fest gewillt, den realen konkreten Gewinn als Besteuerungsmaßstab zu wählen; denn die Besteu-

394 Sonnenfels, Joseph von: Grundsätze, S. 262-267.

395 Eschenmayer, Philipp Christoph Heinrich: Vorschlag zu einem einfachen Steuersystem, S. 30 .

396 Jenetzky, Johannes: System und Entwicklung, S. 225.

397 Ebd., S. $225 \mathrm{ff}$.

398 Schremmer, Eckart: Über „gerechte Steuern“, S. 18.

399 Ebd., S. 18. 
erung des reinen Einkommens war für sie das unbestreitbare Ideal. ${ }^{400}$ Über die praktische verwaltungstechnische Durchführung war man sich nicht im Klaren. Die Gegensätze zwischen formal korrekter äußerlicher Schätzung und der Unmöglichkeit, mit dem zur damaligen Zeit unzureichenden Instrumentarium eine exakte, gerechte Einzelbesteuerung zu erreichen, konnten die Kameralisten nicht lösen. ${ }^{401}$ Der Durchbruch zu einer realen Einzelbesteuerung von konkret erzielten Erträgen erfolgte erst im Laufe des 19. Jahrhunderts.

\subsection{Internationale revolutionäre Theorien und ihr Einfluss auf das steuerpolitische Denken}

\subsubsection{Die Ideale des Liberalismus und die „klassischen Maximen" von Adam Smith}

In Großbritannien machten die Landwirtschaft, der Bergbau, die Industrie sowie der Handel im 18. Jahrhundert beträchtliche Fortschritte, wozu nicht zuletzt die wirtschaftliche Erschließung überseeischer Gebiete beitrug. Mit dieser Entwicklung ging auch die wissenschaftliche Erforschung des Wirtschaftslebens einher. Der Engländer William Petty (1623-1687), der die Arbeitswerttheorie entwickelte und sich mit dem Erfassen ökonomischer Massen- und Durchschnittserscheinungen beschäftigte, sowie der Schotte Adam Smith (1723-1790) waren die herausragenden Nationalökonomen ihrer Zeit. ${ }^{402}$

Adam Smith, der sich zunächst wissenschaftlich mit Moraltheorie und Logik befasste, wandte sich nach einer Reise auf den europäischen Kontinent diesem neuen Wissenschaftsgebiet intensiv zu. Als Moraltheoretiker ging er der Frage nach, was die zivilisierte Gesellschaft freier Menschen zusammenhält, und fragte danach, weshalb Menschen neben ihrem Selbsterhaltungstrieb auch Nächstenliebe und Altruismus entwickeln. Es lag durchaus nahe, dass bei dieser Untersuchung das Interesse von Adam Smith auf die ökonomischen Grundlagen der Gesellschaft gelenkt wurde. Ergebnis seiner langjährigen Studien war 1776 die Publikation eines

400 Kröncke, Claus: Über die Grundsätze, S. 96; Rau, Karl Heinrich: Lehrbuch der politischen Oekonomie, Erste Abtheilung, S. 62 und S. 105.

401 Jenetzky, Johannes: System und Entwicklung, S. 229.

402 Sahm, Reiner: 5000 Jahre Steuern, S. 169. 
mehr als tausendseitigen Manuskripts unter dem Titel „An Inquiry into the Nature and Causes of the Wealth of Nations". 403

Eingehend untersuchte Smith dabei die Position, die dem Staat im Wirtschaftsprozess zukam. Dazu gaben ihm die umfangreiche Tätigkeit des Staates in Großbritannien und die seiner Ansicht nach schädlichen staatlichen Eingriffe in das Wirtschaftsgeschehen besonderen Anlass. Aufgrund seiner Erkenntnisse übte er Kritik an der Wirtschaftstheorie und der wirtschaftpolitischen Praxis der Merkantilisten und Physiokraten und forderte einschneidende Reformen. ${ }^{404}$

Seine wirtschaftspolitischen Empfehlungen lassen sich wie folgt zusammenfassen:

Unter der Bedingung, daß,natürliche Freiheit' in einem Lande herrscht, der moralische Selbstschutz im Volke intakt ist und Wettbewerb und Rechtsordnung das ökonomische Verhalten disziplinieren, sind in der Regel Staatseingriffe in den Wirtschaftsablauf, aber keinesfalls der Staat selbst überflüssig, weil sonst der Wohlstand des Gemeinwesens abnimmt. Laissez faire in diesem Sinne ist natürlich mit schrankenloser Freiheit oder nacktem Egoismus im Wirtschaftsalltag oder mit einem ,Leben ohne Zwänge ebensowenig vereinbar wie mit Einschränkungen des Wettbewerbs, mit Sonderrechten einzelner Gruppen oder Regionen und mit Eingriffen und Vorschriften bei der Übertragung von Eigentumsrechten. ${ }^{405}$

Nach Ansicht von Smith hat der Staat im Wesentlichen Infrastrukturaufgaben zu erfüllen, muss für die Verfügbarkeit öffentlicher Güter in Bildungs- und Gesundheitswesen und für die Verteidigung des Landes Sorge tragen. Diese Aufgaben muss der Staat in einer Marktwirtschaft seinem Wesen nach erfüllen, damit er überhaupt lebensfähig ist und funktionieren kann. ${ }^{406}$

Smith verfasste im fünften und letzten Buch seines Werkes eine genaue Abhandlung über die Besteuerung und über die Staatsausgaben. Dieses Buch enthält auch die bis heute anerkannten Grundsätze der Besteuerung, die immer wieder von Gelehrten und Praktikern aller Länder wiederholt worden sind. John Stuart Mill hat 1848 diesen vier Prinzipien den Namen die „klassischen Maximen“ gegeben und damit deren grundlegende Bedeutung hervorgehoben. ${ }^{407}$

Die erste Maxime enthielt die Norm für die Steuerverteilung:

403 Ebd., S. 169.

404 Ebd., S. 169.

405 Smith, Adam: Der Wohlstand der Nationen, S. LXII f.

406 Recktenwald, Horst Claus: Würdigung des Werkes, in: Smith, Adam, Der Wohlstand der Nationen. Eine Untersuchung seiner Natur und seiner Ursachen (1776), aus dem Englischen übertragen und mit einer umfassenden Würdigung des Gesamtwerkes hrsg. von Horst Claus Recktenwald, 13. Aufl., München 2013, S. XV-LXXIX, S. LXV.

407 Mill, John Stuart: Principles of Political Economy, S. 802. 
Die Bürger eines jeden Landes sollten eigentlich zur Finanzierung der öffentlichen Aufgaben soweit als möglich im Verhältnis zu ihren Fähigkeiten beisteuern, was bedeutet, daß sich ihr Beitrag nach dem Einkommen richten sollte, das sie jeweils unter dem Schutz des Staates erzielen. ${ }^{408}$

Smith entnahm die Norm der Steuerverteilung keinem ethischen Gebot; er sprach nicht von "equity“ (Gerechtigkeit), sondern von „equality“ (Gleichmäßigkeit). Er forderte eine Steuerbelastung, die sich an den Fähigkeiten orientierte, weil diese allein eine Gleichmäßigkeit verbürgte. Diese erste Maxime ist jedoch in der Literatur auf die ethische Ebene projiziert worden, indem man Gleichmäßigkeit mit Gerechtigkeit gleichsetzte. Die Steuerverteilung nach den Fähigkeiten wurde daher aus einem Gerechtigkeitsideal abgeleitet, was dazu geführt hat, dass diese erste Maxime zur anerkannten steuerpolitischen Richtschnur des 19. Jahrhunderts geworden ist. ${ }^{409}$

Die zweite Maxime forderte den Ausschluss der Willkür der Obrigkeit:

Eine Steuer, die jeder einzelne zu zahlen verpflichtet ist, sollte genau und nicht willkürlich festgelegt sein. Der Steuertermin, die Zahlungsform und der zu entrichtende Betrag sollten für den Steuerpflichtigen und jeden anderen klar und offenkundig sein. ${ }^{410}$

Damit hatte Smith dem individualistischen Ideal der Aufklärungszeit - der politischen Freiheit - die Stichworte gegeben, die im 19. Jahrhundert immer wieder benutzt worden sind. ${ }^{411}$ So schlug sich z. B. die Gefahr der Preisgabe der Privatsphäre gegenüber der Obrigkeit im Gesetz wegen Einführung einer Klassensteuer 1820 in Preußen nieder: Die Klassensteuer sollte „Zwischen einer ohne genaues Eindringen in die Vermögensverhältnisse der Pflichtigen nicht ausführbaren und deshalb immer gehässigen Einkommensteuer und einer die Gesamt-Masse aller Einwohner ohne allen Unterschied gleich treffenden Kopfsteuer die Mitte halten. ${ }^{{ }^{4} 12}$

Die dritte Maxime bezog sich auf die Durchführung der Belastung durch die Steuerverwaltung:

Jede Steuer sollte zu einer Zeit oder auf eine Art und Weise erhoben werden, daß die Zahlung der Abgabe dem Pflichtigen am leichtesten fällt. ${ }^{413}$

408 Smith, Adam: Der Wohlstand der Nationen, S. 703.

409 Mann, Fritz Karl: Steuerpolitische Ideale, S. 147 f. und S. 157.

410 Smith, Adam: Der Wohlstand der Nationen, S. 704

411 Mann, Fritz Karl: Steuerpolitische Ideale, S. 227.

412 Gesetz wegen Einführung einer Klassensteuer, gegeben Berlin, den 30ten Mai 1820, zitiert in: Bundesministerium der Finanzen: Neue Ideen und Wege.

413 Smith, Adam: Der Wohlstand der Nationen, S. 704. 
Mit der vierten Maxime hat Smith eine über die Technik der Steuerverwaltung hinausreichende Problematik der Belastung der Steuerbürger dargelegt:

Jede Steuer sollte so erhoben werden, daß sie aus den Taschen der Leute nicht viel mehr nimmt oder heraushält, als sie an Einnahmen in die Kasse des Staates bringt. ${ }^{414}$

Hierzu führte Smith aus, dass auf vier verschiedenen Wegen den Bürgern durch die Steuern mehr weggenommen wird (bzw. man ihnen nicht zukommen lässt), als dem Staat letztlich zufließt. Er begründete dies erstens damit, dass große Erhebungskosten den größeren Teil des Steueraufkommens aufzehren können, zweitens damit, dass eine Abgabe den Erwerbsfleiß der Bevölkerung beeinträchtigen kann. Als dritten Weg führte er diejenigen an, die erfolgslos versuchen, Steuer zu hinterziehen, und dadurch Strafen erleiden, und viertens verwies er auf die verhasste Überprüfung durch den Steuereinnehmer. Die Steuern sollten - so fasste er den Inhalt der Maxime zusammen - den Bürger nicht mehr belasten, als sie dem Staat Nutzen bringen. ${ }^{415}$

Seine im fünften Buch über die Besteuerung und Staatsausgaben vorgetragenen Argumente schlugen sich im 19. Jahrhundert in einer liberalen Weltanschauung nieder und prägten das Denken von liberalen Politikern und Schriftstellern. Smith hat seine Besteuerungsgrundsätze weder gewichtet noch auf Widersprüche hin untersucht und hat aus diesen auch kein ,ideales Steuersystem“ entwickelt, doch spielen sie auch heute noch in Theorie und Praxis eine bedeutende Rolle. ${ }^{416}$

\subsubsection{Der Einfluss der Freiheitsbestrebungen in den amerikanischen Kolonien}

Es waren eigentlich geringfügige Anlässe, die einen weitreichenden gesellschaftlichen Entwicklungsprozess zunächst in Nordamerika und dann auf dem europäischen Kontinent auslösten. Im Jahr 1765 verabschiedete das britische Parlament zwei gegen die Interessen der Menschen in den Kolonien gerichtete Gesetze. Das eine Gesetz, die „Quartering Act“ (die Quartierakte) gab der Regierung das Recht, eine unbegrenzte Anzahl Truppen in die Kolonien zu entsenden und bei der Bevölkerung einzuquartieren. Das andere Gesetz war die „Stamp Act“ (die Stempelsteuerakte),

414 Ebd., S.704.

415 Ebd., S. 705.

416 Ebd., S. LXIX mit dem Hinweis auf das Werk von Neumark, Fritz: Grundsätze gerechter und ökonomisch rationaler Steuerpolitik. 
mit deren Erträgen die britischen Beamten und das Militär in den Kolonien bezahlt werden sollten. Beide Gesetze stießen in den Kolonien auf Ablehnung. ${ }^{417}$

Obgleich die Kolonisten sich nicht grundsätzlich sträubten zu den Staatsausgaben beizutragen, so wollten sie nicht billigen, dass ein Parlament, in dem kein Siedler Sitz und Stimme hatte, darüber befand, dass sie Steuern zu zahlen hatten. Aber die „Stamp Act“, nach der für jede Urkunde und jede Druckschrift bei den Behörden ein Papier erworben werden musste, das nur abgestempelt rechtswirksam war, forderte die Kolonisten zum Widerstand heraus. Die Kolonisten beriefen sich dabei auf das allgemeine Natur- und Menschenrecht. ${ }^{418}$

Nachdem am 1. November 1765 das Stempelgesetz in Kraft getreten war, kam es zu Unruhen. Nun weigerte sich jedermann, Stempelpapiere zu erwerben oder sich an der Steuererhebung zu beteiligen. Die Stempelpapiere wurden vernichtet, Akten der Steuereinnehmer verbrannt und deren Einrichtungen zerstört. ${ }^{419}$

Ein weiterer Steuerplan erfolgte im Jahr 1767; nun wurden u. a. Glaswaren, Papier, Porzellan und Farben mit Abgaben belegt. Die Umsetzung dieses Steuerplanes löste in den amerikanischen Kolonien erneut einen entschiedenen Protest aus. Die Kolonisten verabredeten, keine englischen Waren mehr zu kaufen, solange diese mit einer Abgabe belegt waren. ${ }^{420}$

Unter dem Eindruck der Wirkung, die dieser Widerstand im Mutterland hatte, entschied sich Lord North 1770 für ein anderes Verfahren. Er verzichtete auf die Abgabe im Allgemeinen und belegte lediglich Tee mit einer geringfügigen Steuer. Das geschah in der Absicht, das Besteuerungsrecht Großbritanniens in den Kolonien weiterhin zu wahren, ohne den Handel insgesamt zu gefährden. ${ }^{421}$

Im Dezember 1773 nötigte der Magistrat von Boston den Kapitänen der eingelaufenen Schiffe das Versprechen ab, den Tee, den sie an Bord hatten, nicht an Land bringen zu lassen. Darüber beschwerte sich der Vertreter der Ostindischen Kompanie beim Statthalter, der daraufhin das Löschen des Tees anordnete. Eine Schar von Männern, die sich als Indianer verkleidet hatten, enterten am 18. Dezember 1773 unter Kriegsgeheul die Schiffe und warfen 342 Kisten mit Tee in das Hafenbecken. ${ }^{422}$ Die sogenannte Tea Party setzte eine Bewegung in Gang, die nicht mehr zu stoppen war. Es kam am 19. April 1775 bei Concord und Lexington zu

417 Sahm, Reiner: 5000 Jahre Steuern, S. 177.

418 Ebd., S. $177 \mathrm{f}$.

419 Ebd., S. 181.

420 Schlosser, Friedrich Christoph: F. C. Schlossers Weltgeschichte für das deutsche Volk, Bd. 16, Frankfurt am Main 1854, S. 476.

421 Ebd., S. 476.

422 Ebd., S. 477. 
Gefechten, bei denen die Engländer erhebliche Verluste erlitten. Diese militärische Auseinandersetzung wird als der eigentliche Beginn des nordamerikanischen Freiheitskampfes angesehen. ${ }^{423}$

Es war vor allem die Idee vom naturgegebenen Recht des Menschen auf „Leben, Freiheit und Streben nach Glück“, die Thomas Jefferson, einer der Gründerväter der Vereinigten Staaten von Amerika, vertrat, und die bei vielen Kolonisten auf Zustimmung stieß. Am 4. Juli 1776 nahm der Kongress die von Thomas Jefferson entworfene Unabhängigkeitserklärung an und sagte sich damit vom Mutterland los. In dieser Erklärung wurden erstmals die Menschenrechte formuliert und gesetzlich verankert. Darin heißt es:

Folgende Wahrheiten erachten wir als selbstverständlich: daß alle Menschen gleich geschaffen sind; daß sie von ihrem Schöpfer mit gewissen unveräußerlichen Rechten ausgestattet sind; daß dazu Leben, Freiheit und das Streben nach Glück gehören; daß zur Sicherung dieser Rechte Regierungen unter den Menschen eingerichtet werden, die ihre rechtmäßige Macht aus der Zustimmung der Regierten herleiten.

Ferner wurde in dieser Erklärung ein Widerstandsrecht des Volkes postuliert: „daß, wenn irgendeine Regierungsform sich für diese Zwecke als schädlich erweist, es das Recht des Volkes ist, sie zu ändern oder abzuschaffen und eine neue Regierung einzusetzen. ${ }^{4} 24$

Dem energischen Handeln der amerikanischen Steuerrebellen ist es einerseits mit zu verdanken, dass Bürger nicht ohne Einverständnis mit Steuern belegt werden können, andererseits war jeder Bürger aufgrund seiner geschützten Rechte verpflichtet, seinen Teil zum Wohl der Vereinigten Staaten beizutragen. ${ }^{425}$

\subsubsection{Die liberale Denkweise von 1789 als Fundament neuer steuerpolitischer Ansichten}

In der ersten Hälfte der Achtzigerjahre des 18. Jahrhunderts war die Finanzlage in Frankreich so desolat, dass sich kaum jemand fand, französische Staatsanleihen zu zeichnen. In seiner Ausweglosigkeit unterbreitete der Präsident des königlichen Finanzrats am 8. August 1788 König Ludwig XVI. den Vorschlag, die Generalstän-

423 Ebd., S. 480.

424 Die Unabhängigkeit, Kongreßsitzung vom 4. Juli 1776. Einstimmige Erklärung der dreizehn Vereinigten Staaten von Amerika, in: www. usa.usembassy.de - unabhängigkeit

425 Sahm, Reiner: 5000 Jahre Steuern, S. 189 f. 
de - die Ständeversammlung von Adel, Geistlichkeit und Bürgertum, die seit 1614 noch nicht wieder getagt hatte - für den 1. Mai des nächsten Jahres einzuberufen.

Am 5. Mai 1789 traten die Generalstände zusammen und fassten den folgenden Beschluss: Alle bisher erhobenen Abgaben seien ungesetzmäßig, weil sie nicht von der Nation bewilligt worden seien. ${ }^{426}$ Am 17. Juni 1789 stimmte daraufhin die Nationalversammlung mit überwiegender Mehrheit für ein Dekret, das die Steuererhebung und die Aufrechterhaltung des öffentlichen Schuldendienstes gewährleistete. ${ }^{427} \mathrm{Am} 22$. Juni 1789 fand in Versailles die Kronsitzung statt, die dazu führte, dass den Generalständen das Steuerbewilligungsrecht ebenso zugestanden wurde wie die Garantie der Freiheit der Person und der Presse. Am 9. Juli 1789 erklärten die Vertreter der drei Stände sich zur verfassungsgebenden Nationalversammlung, der Konstituante. ${ }^{428}$

Der König ließ daraufhin Paris mit militärischen Einheiten, die aus ausländischen Söldnern bestanden, umstellen. Dies und weitere Ereignisse mobilisierten die Pariser, und als dann auch noch ein deutsches Dragonerregiment gegen die Demonstrierenden vorgegangen war, wurde in der Nacht zum 13. Juli die Sturmglocke angeschlagen. Am 14. Juli 1789 erstürmten die Pariser die Bastille. ${ }^{429}$

In den Provinzen löste sich in diesen Wochen das staatliche System auf, die Intendanten verließen ihre Posten, was zur Folge hatte, dass im Staatshaushalt keine Steuern mehr eingingen. Die Bevölkerung erwartete die Abschaffung der indirekten Steuern und die strenge Überwachung des Getreidehandels und weigerte sich, Steuern zu zahlen. Im Juli 1789 kam es allerorten zu Bauernerhebungen, in deren Gefolge die Feudalordnung zerstört wurde. ${ }^{430}$

Am Abend des 4. August 1789 tagte die Nationalversammlung und beschloss, alle Steuerprivilegien, die Fron, die "Tote Hand“ und andere persönliche Dienstleistungen abzuschaffen. Des Weiteren sollten die dinglichen Feudalrechte zum Rückkauf freigegeben und auch alle Sonderrechte der Stände, Provinzen und Städte beseitigt werden. Dazu gehörten die Exklusivrechte auf Jagd, Kaninchengehege und

426 Schlosser, Friedrich Christoph: F. C. Schlossers Weltgeschichte für das deutsche Volk, Bd. 17, Frankfurt am Main 1855, S. 173.

427 Ebd., S. $173 \mathrm{f}$.

428 Sahm, Reiner: 5000 Jahre Steuern, S. $217 \mathrm{f}$.

429 Ebd., S. 218.

430 Ebd., S. $218 \mathrm{f}$. 
Taubenschläge. Abgeschafft wurden ferner die Patrimonalgerichtsbarkeit und der Ämterkauf ${ }^{431}$; der Klerus verzichtete auf den Zehnt. ${ }^{432}$

Der höchste Wert der liberalen Denkweise von 1789 war die individuelle Freiheit, die sich schon in der englischen Bill of Rights von 1689 niedergeschlagen hatte. Durch die Freiheitsbestrebungen der Kolonisten in Nordamerika gelangte diese in die amerikanische Verfassung und stellte die erste einklagbare und durchsetzbare Grundrechteordnung dar. Die französischen Soldaten, die mitgeholfen hatten, den Amerikanern die Freiheit zu verschaffen, und die 1783 heimkehrten, waren von den „Söhnen der Freiheit“ angesteckt worden. Mit der Französischen Revolution gelangten die Freiheitsrechte in die Erklärung der Menschen- und Bürgerrechte vom 26. August 1789. Der Entwurf einer Reichsverfassung in der Paulskirche von Frankfurt am Main von 1849 enthielt ebenfalls einen Grundrechtskatalog, die Weimarer Reichsverfassung von 1919 sowie das Grundgesetz von 1949 beinhalten Grundrechtsverbürgungen bzw. Grundrechte. ${ }^{433}$

In all diesen Verfassungen ist auch das Grundrecht der Gleichheit kodifiziert. In der französischen Verfassung vom 3. September 1791 wurde das Prinzip der Allgemeinheit und Gleichmäßigkeit der Besteuerung zum Ausdruck gebracht: „Die Verfassung verbürgt als natürliche und bürgerliche Rechte: [...] 2. daß alle Abgaben auf alle Bürger gleichmäßig unter Berücksichtigung ihrer Vermögensverhältnisse verteilt werden. ${ }^{4334}$

Gleichheit in dem Sinne, dass die Menschen nicht von Natur aus unterschiedliche Rechte und einen unterschiedlichen Status haben, enthielt der Entwurf der Frankfurter Paulskirchenverfassung von 1849 und wurde in Art. 134 (1) der Weimarer Reichsverfassung von 1919 kodifiziert: „Alle Staatsbürger ohne Unterschied tragen im Verhältnis ihrer Mittel zu allen öffentlichen Lasten nach Maßgabe der Gesetze bei." Im Bonner Grundgesetz von 1949 ist der Gleichheitsgrundsatz in Art. 3 (1) verankert: „Alle Menschen sind vor dem Gesetz gleich.“

431 Oswald Spengler hat den Ämterkauf „verschleierte Steuer“ genannt, „die mit furchtbarer Härte allein auf der vornehmen Gesellschaft lastete und zur Aufzehrung ganzer Familienvermögen führte." Spengler, Oswald: Das Verhältnis von Wirtschaft und Steuerpolitik seit 1750. Vortrag gehalten am 19.09.1924 vor dem Verein zur Wahrung der wirtschaftlichen Interessen von Rheinland und Westfalen in Düsseldorf, in: Ders.: Politische Schriften, München 1933, S. 296-310.

432 Sahm, Reiner: 5000 Jahre Steuern, S.219f.

433 Schremmer, Eckart: Über „gerechte Steuern“, S. 12.

434 Aus: http://www.verfassungen.eu/f/fverf91.htm (letzter Zugriff: 22.02.2017). Damit förderte die Französische Revolution ,wirksam die Idee der Allgemeinheit und der Gleichmäßigkeit der Besteuerung nach der Leistungsfähigkeit.“, vgl. Tipke, Klaus: Die Steuerrechtsordnung, S. 543. 
Brüderlichkeit, das 1789 als drittes Grundrecht unabdingbar erklärt wurde, bezeichnet den Zusammenhalt einer Gesellschaft; es ist die Achtung vor dem anderen. ${ }^{435}$ Uneigennützigkeit und der Wille zu helfen, also „soziale Verantwortlichkeit“, schwingen im Begriff der Brüderlichkeit mit. Adolph Wagner entwickelte Ende des 19. Jahrhunderts die Lehre von der sozialen Funktion der Besteuerung, die schrittweise in die Steuerpolitik der Länder eindrang. Die soziale Staatszielbestimmung wurde in Art. 20 Abs. 1 und Art. 28 Abs. 1 im Grundgesetz der Bundesrepublik Deutschland verankert.

\subsection{Zusammenfassung und Fazit}

Mit Beendigung des Dreißigjährigen Krieges begann eine neue Periode: der Sieg des absoluten Staates über den ständischen Staat. Bei den Veränderungen im Steuerund Abgabewesen kam es in erster Linie darauf an, das Einnahmewesen auf eine neue Basis zu stellen. Mit dem Wachsen der Staatsintervention, dem Vordringen der Geldwirtschaft und der Einführung stehender Heere wuchs der Finanzbedarf; die Steuern gediehen zu einer ständigen Zwangsabgabe.

Alle notwendigen Voraussetzungen für ein ergiebiges und geordnetes Abgabesystem fehlten jedoch und die zunehmenden steuerlichen Belastungen gefährdeten die wirtschaftliche Existenz und die persönliche Freiheit der Untertanen. Dieses Versagen des Absolutismus rief eine Gegenbewegung sowie eine neuartige steuerpolitische Idee hervor. Schritt für Schritt drangen naturrechtliche Gedanken in die regierten Kreise und in die politische Opposition vor. Staat und Bürger wurden nunmehr als gleichberechtigte Partner auf steuerlichem Gebiet anerkannt; denn da im Naturzustand alle Menschen frei und gleich sind, gründet sich die Herrschaft auf der Zustimmung der Herrschaftsunterworfenen.

Die Idee der Steuergerechtigkeit trat in den Mittelpunkt der wissenschaftlichen Diskussionen, die Grundsätze der Besteuerung, das Allgemeinheits-, das Gleichmäßigkeits- sowie das Leistungsfähigkeitsprinzip wurden zu politischen Glaubensartikeln. Aus diesem Evolutionsprozess heraus entstanden Grundsätze der Besteuerung, die eine eigenständige Leistung der kameralistischen Finanzwissenschaft waren, die nach Originalität und Folgewirkung ohne Vergleich dastand. Diese „Leitlinien“ stellten im Wesentlichen Gerechtigkeitspostulate, aber auch wirtschafts- und finanzpolitische Erwägungen dar. Heute stimmen diese kamera-

435 Schremmer, Eckart: Über „gerechte Steuern“, S. 12. 
listischen Besteuerungsgrundsätze mit dem geltenden Steuerrecht überein, soweit es sich nicht um Grundsätze handelt, die durch die Zeit überholt sind.

Adam Smiths Werk „Wealth of Nations“ von 1776 gilt als ein grundlegendes Werk der Wirtschaftswissenschaft. Seine im fünften Buch über die Besteuerung und Staatsausgaben vorgetragenen Argumente schlugen sich im 19. Jahrhundert in der liberalen Weltanschauung nieder und prägten das Denken von liberalen Politikern und Schriftstellern.

In den amerikanischen Kolonien setzte sich die allgemeine Auffassung durch, dass sich das Besteuerungsrecht nicht aus einer Staatsgewalt, sondern aus den allgemeinen Menschenrechten ableitet. In der von Thomas Jefferson entworfenen Unabhängigkeitserklärung wurden erstmals die Menschenrechte formuliert und gesetzlich verankert.

Die Freiheitsrechte gelangten in Frankreich in die Erklärung der Menschen- und Bürgerrechte (vom 26. August 1789) - und in der französischen Verfassung (vom 3. September 1791) wurde das Prinzip der Allgemeinheit, der Gleichmäßigkeit und der Leistungsfähigkeit der Besteuerung zum Ausdruck gebracht.

Günter Schmölders hat die Bedeutung der Französischen Revolution für die Geschichte der Besteuerung wie folgt zusammengefasst:

Für die Geschichte der Besteuerung beginnt die Neuzeit im Grunde erst mit der großen Revolution in Frankreich, deren Ursprünge nicht zuletzt in dem ohnmächtigen Hass weiter Kreise der Bevölkerung gegen das Steuersystem des ,Ancien Régime‘ zu suchen sind. [...] der doppelte Grundgedanke, von dem die Steuerreform getragen war, die ,Gleichheit der Provinzen und der Personen vor der Besteuerung`(Vignes), bedeutete eine endgültige Abkehr sowohl von dem Prinzip des Feudalstaates, die Steuerpflicht in erster Linie nach Rang und Stand statt nach ökonomischen Maßstäben zu bemessen, als auch von der regionalen Finanzautonomie und den Steuerprivilegien der Territorialherren; ganz von selbst wurde Frankreich als zentralverwalteter Einzelstaat damit zur Heimstatt der neuzeitlichen Entwicklung der großen Staatssteuern. ${ }^{436}$

\section{Zum Grundsatz der Allgemeinheit der Besteuerung}

Ohne Ausnahme verstanden die Kameralisten unter dem bis heute gültigen Prinzip der Allgemeinheit der Besteuerung die unverrückbare Maßgabe, dass alle Staatsbürger steuerpflichtig sind. Begründung für die allgemeine Pflicht, Steuern zu zahlen, war die Garantie der bürgerlichen Freiheit und der Schutz vor Angriffen von innen und außen.

436 Schmölders, Günter: Geschichte der Besteuerung, S. 621. 
Die frühen Kameralisten sahen in der Akzisebesteuerung den Ansatz eines privilegienfreien Kurses gegenüber dem Adel und dem Klerus, denn mit der Akzise wurden diese Kreise überhaupt erst in die Steuerpflicht einbezogen.

Die späteren Kameralisten haben die Fragwürdigkeit einer solchen einseitigen Verbrauchsbesteuerung erkannt und setzten sich daher für eine durchgehende direkte Besteuerung ein. Ihnen war jedoch bewusst, dass die Finanztechnik auch im beginnenden 19. Jahrhundert noch zu rückständig war, um dem Grundsatz der Steuergleichheit angemessen zu genügen.

Die historisch gewachsenen Steuerfreiheiten lehnten die Kameralisten nahezu einhellig ab. Ihrem Verständnis entsprechend traf den Adel die Verpflichtung, seine nicht mehr ausgeübten Kriegsdienste durch Steuerzahlungen auszugleichen, da das neuzeitliche Kriegswesen aus dem Steueraufkommen bestritten wurde. Der Geistlichkeit wurde jede Berechtigung auf Steuerfreiheit abgesprochen.

\section{Zum Grundsatz der Gleichmäßigkeit der Besteuerung}

Mit dem Gebot der Gleichmäßigkeit forderten die Kameralisten, dass alle Bürger nach gleichen Regeln und nach einem gleichen, in ihrem Verhältnis zum Staate begründeten Maßstab beigezogen werden sollen. Ein Bürger, der - bei gleichen Erträgen - im Verhältnis zu seinen Mitbürgern mehr oder weniger als sie an Steuer zu entrichten habe, ist den Kameralisten zufolge über das rechte Maß belastet bzw. befreit.

Garant für eine effektive Gleichmäßigkeit in der Besteuerung war, dass die Steuern nur vom „Gewinnst“ oder „Ertrag“ genommen werden durften. Aus dieser Erkenntnis heraus entwickelte sich ein eindeutiger Trend von der Vermögensteuer als Substanz- zur Einkommensteuer als Ertragsbesteuerung. Eines der größten Probleme bestand jedoch darin, den „Gewinnst“ zu ermitteln, was aufgrund der mangelnden Buchführungsgrundlagen äußerst problematisch war. Innerhalb des Systems der Ertragsbesteuerung durchlief in der Kameralepoche der Besteuerungsmodus den Weg von der schematisierten hypothetischen Besteuerung über die generalisierte typisierte zur konkret individuellen Besteuerung des Ertrages.

Überwiegend vertraten die Kameralisten den Leitgedanken der harmonisch abgestimmten Steuervielfalt; denn eine gerechte Steuerordnung erforderte die Einheit und Widerspruchsfreiheit einer Mehrzahl von Steuern. Nur eine sinnvolle Kombination mehrerer Steuerarten ist in der Lage, wenigstens tendenziell - dank wechselseitiger Kompensation der Auswirkungen der unterschiedlichen Eigenschaften dieser Steuerarten - der Gesamtheit der Postulate zu entsprechen. 


\section{Zum Prinzip der Besteuerung nach der Leistungsfähigkeit}

Es waren die großen politischen Autoren der Aufklärungszeit, die zur Mitte des 18. Jahrhunderts mit der Besteuerung nach der individuellen Leistungsfähigkeit ein eigenständiges Prinzip ausformulierten und mit den Vorschlägen der Steuerprogression und der Freilassung des Existenzminimums die Öffentlichkeit gewonnen haben. Die neue geistige Haltung strahlte auf alle Bereiche der Steuerpolitik aus, dem Glauben an die Naturordnung folgte ein völliger Meinungsumschwung: Die allgemein anerkannte Assekuranztheorie führte zu der Überzeugung, dass der sicherste Maßstab für die Steuerhöhe das Vermögen, der Ertrag und das Einkommen seien. Damit führten die vom Naturrecht ausgehenden Überlegungen zwangsläufig zu einer Bevorzugung der direkten Steuern.

Obwohl die überwältigende Mehrheit die direkte Besteuerung der indirekten Besteuerung vorzog, waren viele Autoren nicht bereit, diesem Ziel zu folgen, denn die Finanztechnik zu Beginn des 18. Jahrhunderts und die Bemessungsgrundlagen steuerlicher Leistungsfähigkeit waren zu rückständig bzw. zu schwer zu erfassen, um der Gerechtigkeit zu genügen.

Proportionale und progressive Besteuerung wurden kontrovers diskutiert. Der deutsche Kameralist Justi zeigte an einem Beispiel, wie unbefriedigend die proportionale Besteuerung ist, bei der die Reichen nicht entsprechend erfasst werden und die ganze Last auf den Schultern des Mittelstandes ruhe. Die Idee einer progressiven Besteuerung gewann in der Argumentation in dem Maße an Gewicht, in dem die Industrialisierung voranschritt; am Ende der Kameralzeit war der Gedanke der progressiven Besteuerung vorherrschend.

Unabhängig von der Kritik an den historisch begründeten Steuerfreiheiten entwickelten die kameralistischen Autoren spezielle Steuerfreiheiten - wie z.B. für Hospitäler, Armenhäuser etc. -, die wegen der wirtschaftlichen Notwendigkeit gefordert wurden. Arme Leute galten als nicht belastbar und seien daher von jeder steuerlichen Belastung freizustellen. „Erhalt des Notbedarfs“, „Schonung der Armen“ und die Besteuerung erst ab "Überschreiten des Existenzminimums“ sind die Stichworte, die die Untergrenze der Besteuerung und die Festlegung der absoluten Belastungsgrenze markieren.

\section{Zum Redistributionspostulat oder der Grundsatz der steuerlichen Umverteilung von Einkommen und Vermögen}

Gestützt auf die Überzeugung, dass der Staat in der Regel kein Unrecht tun kann, schlugen die Kameralisten ab der Periode des Hochkameralismus Eingriffe in die private Vermögenssphäre durch steuerliche Maßnahmen vor. Die Steuern sollten 
Luxus und Verschwendung unterdrücken, und die Wirtschaft sollte durch steuerliche Eingriffe je nach Bedarf eingeschränkt oder gefördert werden.

Jean-Jacques Rousseau verwies darauf, dass das physische Existenzminimum bei allen Menschen gleich sei, weshalb das Lebensnotwendige steuerfrei bleiben müsse; hingegen könne das Überflüssige im Notfall ganz weggesteuert werden. Innerhalb einer Sozialphilosophie, die in der Ungleichheit die Ursache allen menschlichen Elends erblickte, war diese Argumentation nur folgerichtig.

Dieses Buch wird unter der Creative Commons Namensnennung 4.0 International Lizenz (http://creativecommons.org/licenses/by/4.0/deed.de) veröffentlicht, welche die Nutzung, Vervielfältigung, Bearbeitung, Verbreitung und Wiedergabe in jeglichem Medium und Format erlaubt, sofern Sie den/die ursprünglichen Autor(en) und die Quelle ordnungsgemäß nennen, einen Link zur Creative Commons Lizenz beifügen und angeben, ob Änderungen vorgenommen wurden.

Die in diesem Buch enthaltenen Bilder und sonstiges Drittmaterial unterliegen ebenfalls der genannten Creative Commons Lizenz, sofern sich aus der Abbildungslegende nichts anderes ergibt. Sofern das betreffende Material nicht unter der genannten Creative Commons Lizenz steht und die betreffende Handlung nicht nach gesetzlichen Vorschriften erlaubt ist, ist für die oben aufgeführten Weiterverwendungen des Materials die Einwilligung des jeweiligen Rechteinhabers einzuholen.

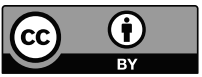

